Russow, Edmund Zur Anatomie resp.physiologisehen vergleichenden Anatomie der Torfmoos Dorpat, 1887. 





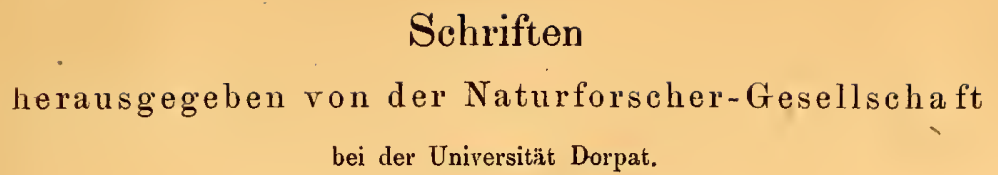

III.

\section{Zur Anatomie}

resp. physiologischen und vergleichenden Anatomie

der

\section{Torfmoose}

von

\section{Dr. Edmund Russow}

ord. Professor der Botanik an der Universität Dorpat.

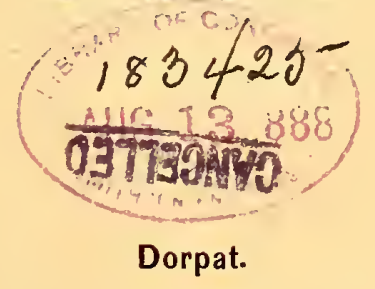

Druck von C. Mattiesen.

1887. 



\section{Zur \\ Feier des Tages}

an welchem vor

\section{fünfzig Jahren}

\section{Dr. Alexander Graf Keyserling}

seine erste wissenschaftliche Arbeit veröffentlichte

widmet

die Dorpater Naturforscher-Gesellschaft

diese Abhandlung

\section{dem hochverdienten Jubilar}

ihrem Ehrenmitgliede.

$D$ e $n$ 27. D e c e m b e r 1887 

Tritt uns im äusseren wie inneren Bau der Gewächse mehr oder weniger ausgesprochen eine Anpassung an die äusseren Lebensbedingungen entgegen, so möchte es doch nur wenige Gewächsgruppen geben, bei denen in so augenfälliger Weise wie bei den Torfmoosen die Anpassung an äussere Verhältnisse sich in der Architektonik dieser Gewächse, als ganz besonders die Planmässigkeit der inneren Construction bis auf die letzten Bausteine, die einzelnen Zellen und deren Theile, in Bezug auf die physiologische Leistung, sich ausspricht.

Es weichen die Torfmoose von allen Bryophyten sowohl im architektonischen, wie besonders im anatomischen Bau weit ab und bilden eine geschlossene, schon äusserlich leicht kenntliche, innerlich in noch höherem Grade differente, überaus scharf umschriebene, natürliche Gruppe, wie wir deren nur wenige im Pflanzenreiche antreffen. Daher ist es verständlich, dass diese Pflanzenfamilie sowohl in morphologischer als anatomischer Beziehung vielfach untersucht worden und dass sich mit dem Studium des inneren Baus nicht nur Sphagnologen, sondern auch zahlreiche und hervorragende Phytotomen beschäftigt, welchen letzteren wir in erster Linie die Kenntniss vom inneren Bau dieser merkwürdigen Gewächse wie deren Entwickelungsgeschichte zu verdanken haben. Ich brauche nur hier an Namen wie Moldenhawer, Meyen, H. v. Mohl, Nägeli, $\mathrm{H}$ ofmeister zu erinnern. W. Ph. Schimper ${ }^{1}$ ) hat in seiner 1858 erschienenen, berühmten Monographie der Sphagna alles bis dahin über Morphologie und Anatomie der Sphagna Bekannte zusammengefasst, und dasselbe mit den Ergebnissen seiner eigenen Untersuchungen verwebend, in einem anschaulichen Bilde dargestellt; daher sei hier bezüglich der ganzen älteren SphagnumLitteratur, soweit sie die Anatomie betrifft, auf das genannte Werk Schimpers verwiesen.

Im Jahre 1865 veröffentlichte ich eine Abhandlung ${ }^{2}$ ) in welcher einige, $\mathrm{Sch}$ imper und seinen Vorgängern entgangene, anatomische Einzelheiten

1) Versuch einer Entwickelungsgeschichte der Torfmoose und einer Monographie der in Europa vorkommenden Arten dieser Gattung. Stuttgart, 1858, m. 27 Tafeln. Folio.

2) Beiträge zur Kenntniss der Torfmoose. m. 5 Tafeln, im Archiv f. d. Naturkunde Liv-, Estund Kurlands. Dorpat 1865 . gr. $8^{\circ}$. 
mitgetheilt und abgebildet wurden, von denen hier der Nachweis weit verbreiteter Resorbtionserscheinungen an der Membran der hyalinen Zellen in den Astund Stengelblättern zahlreicher Arten, wie die Entdeckung der merkwürdigen „Kammfasern“ in den Hyalinzellen der Astblätter von Sph. imbricatum Hornsch. (Sph. Austini Sulliv.) hervorgehoben sein mag. Im Jahre 1868 fand Lindberg bei einigen Formen des Sph. cymbifolium Ehrh. an den Wänden der Hyalinzellen, soweit diese mit den Chlorophyllzellen zusammenstossen, kleine, dicht gestellte Papillen oder spitze Wärzchen, welche den betreffenden Wandstücken, bei 500-600facher Vergrösserung; das Ausehen von mit Mehl oder Gries, bald mehr, bald weniger dicht bestreuten Flächen ertheilen. Der Entdecker fasste die betreffenden Formen unter dem Namen Sph. papillosum, als Art, zusammen. Dieselbe Erscheinung wurde von demselben Forscher einige Jahre später auch bei Sph. squarrosum und teres entdeckt und es mag gleich hier hinzugefügt werden, dass ich kürzlich bei $S p h$. Wulfianum, und hiervon unabhängig anch W a r l storf bei derselben Art, die erwähnten Papillen gefunden.

Phytotomen haben sich meines Wissens nach $\cdot \mathrm{Hug}_{\mathrm{g}} 0 \mathrm{v} 0 \mathrm{n}$ M $0 \mathrm{hl}$ und $\mathrm{S}$ e h a c h t nicht speciell mit der Uutersuchung von Torfmoosen beschäftigt und da im Laufe dieser Zeit die Mikroscopie in Folge der sehr verbesserten optischen Hilfsmittel wesentliche F'ortschritte gemacht, ist es erklärlich, das manche allgemein verbreitete Erscheinungen, welche nur mit Hilfe der bestell uns heute zu Gebote stehenden Mikroscope mit Sicherheit erkannt werden können, bisher entweder gänzlich übersehen worden oder keine richtige Deutung gefunden haben.

Seit bald zwei Jahren habe ich das von mir vor mehr als 20 Jahren zuletzt betriebene Studium der Torfmoose, in systematischer Beziehung, wieder aufgenommen und bin, da die sichere Unterscheidung der Arten oft nur durch die eingehendste mikroscopische Untersuchung, bei Anwendung sehr starker Vergrösserungen, ermöglicht wird, in Folge Anwendung besserer Mikroscope, als sie mir damals zu Gebote standen und wohl noch mehr in Folge gereifterer Erfahrung anf dem Gebiet der Pphytotomie überhaupt, auf manche, früher von mir. gänzlich übersehene Erscheinungen gestossen, oder habe erst jetzt eine richtige Deutung gefunden für Erscheinungen, welche mir zwar schon damals auffielen, die ich mir aber zu erklären nicht vermochte oder die ich unrichtig auffasste.

Auf folgenden Blättern beabsichtige ich diese, unsere Kenntniss von der Anatomie der Torfmoose erweiternden neuen Einzelheiten, im Zusammenhange mit dem bereits Bekannten, aus physiologischem Gesichtspunkt zu behandeln, wodurch, wie ich hoffe, manches neue Licht auf längst Bekanntes fallen wird und wir wenig oder garnicht beachtete Erscheinungen als nothwendig oder dọch wenigstens vortheilhaft für das Bestehen des Ganzen erkennen werden. SChliesslich will ich es versuchen die gewonnenen Resultate, mehr andeutend als ausführend, im Interesse der Systematik zu verwerthen, da ich bald an einem an- 
deren Orte eingehendere Beiträge zur Systematik der Torfmoose zu liefern gedenke.

Den architectonischen wie anatomischen Bau der Torfmoose in den Hauptzügen beim Leser als bekannt voraussetzend, halte ich es im Interesse des richtigen Verständnisses der mitzutheilenden Thatsachen für zweckdienlich, zunächst an einige bekannte biologische Verhältnisse der Torfmoose zu erinnern.

Offenbar hat die Natur die Torfmoose geschaffen, um in nördlichen Breiten oder im Hochgebirge südlicher Zonen, wo das Maass von Feuchtigkeit gross, das der Würme aber gering ist, sanfte Bodeneinsenkuigen von kleiner bis sehr grosser Ausdehnung, welche sonst nur Wasserspiegel darbieten würden, mit höherem Pflanzenleben, und zwar Luftpflanzen, zu besiedeln, dadurch reichlichere organische Substanz zu produciren und ein höheres Thierleben zu ermöglichen. Zu dem Zweck mussten wasserliebende Geirächse construirt werden mit der Befähigung, bei selbständiger Assimilation, Wasser leicht aufzunehmen, sich gleichsam wie ein Schwamm voll Wasser zu saugen und dieses nöthigenfalls zurückzuhalten oder nur schwer verdunsten zu lassen, somit hierdurch einen relativ festen Boden, ein schwammiges Polster zu schaffen, auf welchem zahlreiche Luftpflanzen wie: Cyperaceen, Juncaceen, Ericaceen und selbst grössere Holzgewächse und mithin auch zahlreiche Luftthiere gedeihen können. Dieser Zweck ist sehr vollkommen in den unbegrenzt an der Spitze fortwachsenden, selbständig assimilirenden, aus einem bewunderungswürdigen System feinster Capillaren und kohlensäure assimilirender Zellen zusammengesetzten Organismen erreicht worden, welche wir Torfmoose nennen.

Den beiden angedeuteten Hauptaufgaben entsprechend, nämlich Wasser aufzusaugen wie ein Schwamm und organische Substanz zll produciren, finden wir die Hauptmasse der vegetativen Organe der Torfmoose, die Blätter, construirt aus $z$ w e i erle i Elementen, von denen die einen, die Chlorophyll führenden Zellen, das assimilatorische oder ernährungsphysiologische System darstellen, während die anderen, die hyalinen Zellen, das mechanisch wirksame System, gleichsam das Pumprerk oder Wassersaugungssystem bilden. Diese beiden Zellensysteme sind mit einander zu einem Maschenwerk vereinigt, in welchem bekanntlich die Fäden von den relativ langen und schmalen, im Querschnitt dreieckigen oder trapezoidischen, quadratischen oder rectangulären oder linsenförmigen Chlorophyllzellen gebildet werden, während die rhombischen bis gestreckt sechsseitigen und schwach S-förmig gebogenen Maschen von den durchlöcherten Faser- oder Hyalinzellen eingenommen werden (Fig. 2, Taf. I). Entweder sind die Chlorophyllzellen, wenn ihr Querschnitt linsenförmig ist, vollständig von delı Hyalinzellen umschlossen (Fig. 53, 56-63) oder es ist eine Wandfläche der Chlorophyllzellen frei, bei dreieckigem Querschnitt, wobei sie von der Innen(Fig. 10, 11, 12) oder Aussenfläche des Blattes (Fig. 25-28 u. 30) sich zwischen die Faserzellen gleichsam einkeilen; oder zwei Wandflächen der Chlo- 
rophyllzellen sind frei und in diesem Fall sind die Hyalinzellen vollkommen von einander getrennt; im letzteren Fall ist der Querschnitt der Chlorophyllzellen trapezoidisch (Fig. 15, 23, 40) oder rechteckig (Fig. 13, 14, 32, 46) oder tonnenförmig (Fig. 31, 33, 39, 50).

Den Ansprüchen an Festigkeit und Steifheit wird genügt von Seiten der Chlorophyllzellen durch deren relative Dickwandigkeit wie durch den Turgor, während die früh absterbenden, turgorfreien, äussert dünnwandigen, nur durch ihr Hautgerüst wirksamen und bedeutungsvollen Hyalinzellen, bei möglichst geringem Aufwande von Material, durch Aussteifung vermittelst ring- oder schraubenförmig verlaufender Leisten oder Fasern, die erforderliche Festigkeit erlangen.

Die Ansprüche an Festigkeit sind aber nicht in der ganzen Ausdehnung des Blattes dieselben, auch nicht bei den Blättern der abstehenden und herabhängenden Aeste gleich.

Die Astblätter sind bekanntlich dachziegelartig über einander gelegt, derart, dass etwa das oberste, mehr oder weniger zugespitzte Drittel des Blattes frei hervorragt und mehr oder weniger absteht, während die untere Hälfte gedeckt wird. Demnach steht zu erwarten, dass die obere Hälfte, zumal die frei abstehende Spitze der Blätter abstehender Aeste, fester gebaut sein muss als die untere Hälfte, was in der That der Fall ist, während eine erhebliche Verschiedenheit in dem Bau der oberen und unteren Blatthälfte bei den Blättern der herabhängenden Aeste nicht besteht, welche allseitig geschützt sind; desgleichen bei den Blättern der lang herabhängenden Enden abstehender Aeste, die gleichfalls geschützt sind gegen das Aufschlagen von Regentropfen oder den Anprall fallenden und bewegten Laubes benachbarter Gewächse, oder gegen die Verletzungen von Seiten kriechender Thiere.

In welchem Maasse das umgebende Medium den anatomischen Bau beeinflusst, tritt uns deutlich beim Vergleich der untergetauchten Formen mit den zwar Wasser liebenden, aber zeitweilig von Luft umgebenen Formen der Cuspidatum-Gruppe entgegen. Bei ersteren schwinden häufig die Fasern in den Hyalinzellen, oder sie stehen in grösseren Abständen von einander und sind zarter ausgebildet, und was noch auffallender, es bildet sich oft weitaus der grösste Theil der Zellen des Blattgewebes zu chlorophyllführenden aus, so dass die characteristische Zusammensetzung des Sphagnumblattes, aus ernährungsphysiologischen und mechanısch wirksamen Zellen, in der grösseren Ausdehnung des Blattes vermisst wird 1). Bei den nicht untergetauchten Cuspidatis zeigen die Blätter den gewöhnlichen Bau mit sehr reichlicher und starker Faserbildung in den Hyalinzellen, weichen aber von den übrigen, weniger an reichliches Wasser gebundenen Arten durch die Lochbildung in den Wänden der Hyalinzellen ab, worüber

1) Vrgl. E. Russow, Beiträge z. Kennt. d. Torfmoose, p. 60 wo eine derartige auffallende Wasserform beschrieben ist. 
wir später näher sprechen werden. Dass bei den vorherrschend im Wasser lebenden Formen die Aussteifung der Hyalinzellen durch Fasern nicht erforderlich ist, sehen wir an dem amerikanischen Sph. macrophyllum Bernh., das in Bezug auf Porenbildung mit den Cuspidatis insofern übereinstimmt, als auch hier die Löcher von keinem Ringwall umgeben sind.

Sehen wir uns nun die Einrichtungen näher an: welche zum Zweck der grösseren Festigung der Blattspitze getroffen sind.

Ueberblicken wir die Fläche eines ausgebreiteten Blattes, das wir der unteren Hälfte eines abstehenden Astes entnommen haben (nur von solchen ist zunächst die Rede), so wird uns zunächst die bedeutende Grössenabnahme der Zellen von der Basis zur Spitze des Blattes in die Augen springen, ebenso die Zunahme der Einrollung der Blattränder zur Spitze hil. Bei stärkerer Vergrösserung werden wir, zumal am Querschnitt, wahrnehınen, dass die Wände der sehr viel kleineren Zellen, sowohl der Chlorophyll- als Hyalinzellen, im oberen Drittel des Blattes sehr viel stärker verdickt sind als in der unteren Hälfte, und dass die Fasern der kleineren Hyalinzellen, namentlich in der AcutifoliumGruppe, stärker (in der Aufsicht breiter) sind als die der grősseren Hyalinzellen in der unteren Blatthälfte. Während die Membran der letztgenannten Zellen kaum messbare Dicke besitzt, erreicht sie bei den Zellen der oberen Blatthälfte etwa ein Mikron und darüber.

Dass die Festigkeit, d. h. die Widerstandsfähigkeit einer Zelle gegen Druck und Biegung, bei gleicher Wanddicke, mit Abnahme der Grösse entsprechend zunimmt, ist ein in der Gewebelehre. längst beachtetes Moment. Da nun hier mit der Grössenabnahme der Zellen eine Zunahme ihrer Wanddicke verbunden ist, so wird dadurch die Festigkeit natürlich ganz ausserordentlich erhōht. Die Biegungsfestigkeit des ganzen Blattes wird gesteigert durch die Einrollung der Ränder, zumal an der Blattspitze, wo nicht selten die Ränder über einander greifen, so dass das Blatt in einen Trichter ausläuft. Der Rand des Blattes ist durch einen bald breiteren, bald schmäleren, aus derbwandigen, englichtigen und gestreckten Zellen gebildeten Saum (margo) gegen Einreissen geschütat.

Eine nähere Auseinandersetzung erheischt

\section{die Aussteifung der Hyalinzellen,}

da bisher ein wichtiges Moment in Bezug auf die Faserbildung übersehen, oder doch wenigstens nicht erkannt worden ist.

Bis vor kurzem habe ich mir, wie es wohl bisher allgemein von den Phytotomen und Sphagnologen geschehen, die Ring- wie Spiralfasern an den Wänden der Hyalinzellen vorgestellt als nur wenig in das Lumen der Zelle hineinragende Leisten, von etwa halbkreisförmigem oder quadratischem Querschnitt, mit etwas abgerundeten Kanten. Trifft das für die Fasern in der unteren Blatt- 
hälfte oder in den Rindenzellen der Cymbifolium-Gruppe meist zu, so ist doch in der oberen Blatthälfte, zumal gegen die Spitze des Blattes, die Form der Fasern eine ganz andere. Es sind bald $\mathrm{mehr}$, bald wenjger breite Platten oder Bänder, die mit ihrer scharfen Kante der Zellmembran rechtwinklig ansitzen.

Eine derartige Faserbildung ist mir im übrigen Pflanzenreich nur bei dell IV a m millárien begegnet, wo bekanntlich die dünnwandigen Gefässe des Holzes von ringförmigen oder schraubig gewundenen Platten ausgesteift werden ${ }^{1}$ ).

Ein Blick auf die Abbildungen der Blattquerschnitte auf den beigefügton Tafeln 2-5 lässt sogleich diese, in das Lumen der Hyalinzellen mehr oder weniger tief hineinragenden Ring- und Spiralplatten erkennen (durch leichte Tönung sind die Platten kenntlicher gemacht worden). Es ist eine so auffallende Erscheinung, dass unwillkürlich der Leser die Frage aufwerfen wird, wie ist es möglich, dass eine so augenfällige Bildung sich bisher der Wahrnehmung entzogen? Es sind nun allerdings diese Platten gesehen, ja selbst abgebildet, aber $\mathrm{nicht}$ erkannt worden. Unter den zahlreichen Abbildungen von Blattquerschnitten, die Schimper in seiner genannten Monographie giebt, findet sich eine, aber auch nur e in e Abbildung, auf Taf. XXVII (in der Mitte unten, mit s, 4 bezeichnet) von $S p h$. subsecundum, vair. contortum, an der wir mitten im Lumen der zwei Hyalinzellen einen quer-ovalen, ringförmigen Contour wahrnehmen, doch ist diese Erscheinung weder in der Tafelerkläruıg noch im Texte auseinandergesetzt. Unter den Abbildungen der Blattquerschnitte von Schliephacke, welche Warnstorf seinen ,sphagnologischen Rückblicken“ 2) beigegeben, sind an der Abbildung von Sph. cyclophyllum, zu beiden Seiten der Chlorophyllzellen, schattirte, meniskusförmige Partien gezeichnet, welche auch hier, weder im Texte, noch in der Figurenerklärung eine Besprechung erfahren. Diese Menisken sind nichts Anderes als der Ausdruck der hier wie bei Sph. rigidum und aculeatum $W$. vorkommenden, eigenthümlichen Ringfasern, welche meist nur an dem Theile der Wand, welche mit der der benachbarten Hyalinzelle verwachsen ist, plattenförmig verbreitert sind.

Mir sind bereits vor 24 Jahren bei der Untersuchung der Torfmoosblatter am Quersehnitt die beregten Ringplatten, oder vielmehr die rundlich-ovalen Contouren im Lumen der Hyalinzellen, aufgefallen, doch glaubte ich diese Contouren, da sie nicht in allen Zellen und meist nur an Schnitten, welche die obere Blatthälfte getroffen hatten, sichtbar waren, als Schnittränder der am stark verjüngten Ende getroffenen Hyalinzellen auffassen zu müssen. Die damals von mir untersuchten Schnitte waren ausserordentlich dünn und daher erklärt sich die Abwesenheit der Ringplatten in zahlreichen Zellen. Jetzt, wo ich dickere QuerFig. $52, \mathrm{~B}$

1) Cfr. Schleide n, Grundzüge der Botanik 3, und 4. Auflage 1849 u. 1861. pag. 187.

2) Flora, 1884. Taf. V, Fig. 1. 
schnitte, zumal von Sph. cuspidatum, untersuchte und in jeder Hyalinzelle die Ringplatten wahrnahm, musste ich natürlich an der Richtigkeit meiner alten Auffassung zweifelhaft werden. Die nähere Untersuchung des fraglichen Gegen-' standes ergab Folgendes.

Die Ring- und Spiralfasern in den Blättern der abstehenden Aeste sämmtlichor Arten aus der Sectio Fusphagnum Lindbg und Hemitheca Lindbg, sind in der oberen Hälfte der Blätter mehr oder weniger tief bis sehr tief in's Lumen der Hyalinzellen hineinragende Ring- oder Schraubenplatten, deren Breite (d. $h$. der senkrecht zur Zellmembran orientirte Durchmesser) von der Spitze gegen die Basis des Blattes, absolut nicht sehr beträchtlich, relativ (im Verhältniss zum Querschnitt der Zelle) aber sehr auffallend àbnimmt, (vergl. Fig. 17 ind 20, 12 ․ 14,34 u. 33) um in der unteren, bedeckten Hälfte des Blattes meist als Fasern von etwa quadratischem Querschnitt, zu verlaufen; bei den cuspidatis und subsecundis ist auch in der unteren Blatthälfte meist die Plattenform noch deutlich ausgeprägt. Besonders breit sind die Ringplatten bei Sph. $P y$ laiei, zumal im oberen Drittel des Blattes, wo man die Hyalinzellen als, durch zahlreiche, in der Mitte eng perforirte Querscheidewände, septirt bezeichnen könnte. Fig. 34 u. 35 . Wie mir scheint, hat bereits Li mpricht ') hier den Sachverhalt richtig erkannt wenn er sagt: „Hyaline Zellen mit zahlreichen Ringfasern, die nach innen so stark vorspringen, dass ihr rundes Lumen im ausg'ebreiteten Blatte täuschend Poren längs der Zellmitte ähnlich sieht." Fast dasselbe gilt auch von den Hyalinzellen in der Blattspitze der acutifolia, cuspidata und subsecunda (Fig. 17 u. 22). Bei dell acutifoliis, zumal bei Sph. Warnstorfi m. und fuscum Klingg. und bei Sph. Wulfianum Girg. erscheint der in's Lumen hineinragende Rand der Ringplatten (namentlich in der Blattspitze) doppelt contourirt (Taf. I, Fig. 7 und Taf. II, Fig. 10 u. 18) was daher rührt, dass der Rand gefurcht und auseinandergespreizt ist, wie Fig. 5a zeigt, die den Durchschnitt einer Hyalinzelle aus der Blattspitze, parallel zur Oberfläche geführt, darstellt; hierdurch wird natürlich die Biegungsfestigkeit der Ringplatte nicht unwesentlich erhöht.

Die Ringplatten verlaufen nicht selten schief zur Längsaxe der Zelle; solche Platten werden natürlich, wenn der Querschnitt die Zelle rechtwinklig zur Längsaxe getroffen und dabei sehr dünn ist, zum Theil abgeschnitten werden und als grösseres oder kleineres Bruchstück eines Ringes erscheinen, vergl. Fig. 7, Taf. I und 11, 13 u. 23; dasselbe gilt natürlich von den Spiralplatten.

Die Plattenform der Ring- wie Spiralleisten findet selbstverständlich auch in der Aufsicht eines Blattes, bei mittlerer Einstellung anf die Hyalinzellen, ihren optischen Ausdruck, und somit hätte man schon längst, auch ohne Herstellung ron Querschnitten, nur durch den Anblick der Hyalinzellen im optischen

1) Rabenhorst's Kryptogamenflora, Bd. IV, Lief. 3. pag. 135. 1886. 
.Längsschnitt, von der Natur der „Fasern“ eine richtige Vorstellung gewinnen müssen, wenn man nicht von der vorgefassten Ansicht ausgegangen wäre, dass die „Fasern“ dünne Leisten von etwa halbkreisförmigem oder quadratischem Querschnitt, und in der oberen Blatthälfte ebenso wie in der. unteren gebildet seien. Es erscheint nämlich jede Faser in den Hyalinzellen der Blattspitze, bei mittlerer Einstellung der Zellen, (zumal bei den acutifoliis und cuspidatis) wie aus drei Stücken zusammengesetzt (Fig. 1 u. 2), oder mit anderen Worten, in der Mitte jeder Faser erblicken wir zwei, bald einander, bald den Chlorophyllzellen mehr genäherte Querstreifen, die häufig gekrümmt, und zwar nach aussen, d. h. "zu den Chlorophyllzellen hin convex, vèrlaufen, Fig. 1. Das Mittelstück erscheint wie beschattet und an den (quer zur Längsaxe der Zelle verlaufenden) Rändern etwas verwaschen contourirt, während die beiden Seitenstücke hell erleuchtet und sehr scharf contourirt sich projiciren. Das dunklere Mittelstück ist natürlich nichts Anderes, als der optische Querschnitt des ovalen Loches in der Mitte der Ringplatte. Ist der Innenrand dieser Platte gefurcht, so erblickt man im beschatteten Mittelstück einen dunklen Doppelcontour, parallel den freien Rändern, Fig. 1b, nämlich die Furche in der Aufsicht. Bereits vor 24 Jahren ist mir diese Dreitheilung der Fasern in den Spitzen der Astblätter sehr aufgefallen und hat mir viel Kopfbrechen verursacht. Ich vermochte mir die Ursache der Erscheinung nicht befriedigend zu erklären, mich mit der Mangelhaftigkeit meines Mikroscops tröstend, und da ich weder bei Schimper, noch Mohl oder Meyen diese Erscheinung erwähńt fand, glaubte ich es mit einer optischen Täuschung zu thun. zu haben, durch derẹn Besprechung ich meine Unerfahrenheit vielleicht zu sehr zu documentiren fürchtete.

Noch einer Erscheinung möchte ich hier erwähnen, welche ich mir bis zur Entdeckung der Ringplatten nicht zu erklären vermochte und geneigt war in das Gebiet der optischen Täuschungen zu verweisen. Blickt man auf die, durch Krümmungen und Faltungen der Blattfläche, mehr oder weniger geneigt zur Sehaxe aufgerichteten Hyalinzellen der Blätter von Sph. cuspidatum und subsecundum, so nimmt man in der Mitte der Hyalinzellen, ganz regelmässig zwischen je zwei Fasern, querovale Ringe wahr, welche zunächst den Eindruck von Löchern in der Wand der Hyalinzëlle hervorrufen, bei veränderter Einstellung aber sich bald von den gewöhnlichen Löchern als durchaus verschieden erweisen. Es sind diese querovalen Ringe eben nichts Anderes, als die perspectivisch verkürzten, kreisförmigen oder längsovalen Innenränder der Ringplatten. Ich vermuthe, dass dieselbe Erscheinung von Limpricht bei Sph. Pylaiei gesehen worden, denn ich wüsste keine andere Deutung für die oben citirte Angabe.

Bei spiraligem Verlauf der Platten lässt sich an schief aufgerichteten Hyalinzellen, durch Verạnderung der Einstellung, sehr schön die schraubig gewundene Platte in ihrer ganzen Ausdehnung erkennen. 
Das tiefe Hineinragen der Ring- und Spiralfasern in das Lumen der Hyalinzellen, zumal in den Blattspitzen, wird sehr augenfällig, wenn das Lumen der Hyalinzellen nicht mit Wasser, sondern mit Luft erfüllt ist. Das fast schwarze Zelllumen erscheint dann tief gefurcht oder eingeschnürt," durch helle, einander gegenüberstehende Streifen, zwischen deren Enden schmale, aschgraue Brücken die Verbindung zwischen den schwarzen perlenartigen Partien herstellen. $\mathrm{Zu}$ ëner derartigen Beobachtung eignet sich ganz besonders Sph. Pylaiei, weil wegen des fast gänzlichen Mangels an Löchern in der Membran der Hyalinzellen, in letztere Wasser nur sehr langsam und schwer eindringt, wenn die Blätter vorher ausgetrocknet waren. Für die Plattennatur der Ring- und Spiralfasern spricht endlich noch das Verhalten und Gebahren der in die Hyalinzellen eingeschlüpften Infusorien.

Dem Mitgetheilten zufolge ist an der platten- oder bandförmigen Gestalt der Ring- und Spiralfasern in der oberen Hälfte der Astblätter wohl nicht zu zweifeln und es ist der Zweck dieser Einrichtung leicht einzusehen. Die Aussteifung der Hyalinzellen wird durch breite Ring- oder Spiralplatten, die mit ihrer scharfen Kante der Membran aufsitzen, bei Sparung von Material, in viel höherem Grade erzielt als durch Fasern von quadratischem Querschnitt, bei gleicher Masse des aufgewandten Materials: Offenbar ist aber durch diese Einrichtung nicht nur eine sehr zweckmässige Aussteifung der einzelnen Zellen erreicht, sondern auch einer Verschiebung der Hyalinzellen gegen die Chlorophyllzellen und somit einer Einknickung der Blattfläche vorgebeugt. Beachten wir den Umstand, dass in den vier Ecken der Hyalinzellen, dort wo die freien Aussenwände derselben mit den Chlorophy:lzellen einen fast rechten Winkel bilden, die Ringplatten den grössten Durchmesser besitzen (besonders auffallend in Fig. $22,23,34$ u. 43) so ist der Schluss berechtigt, dass durch die getroffene Einrichtung eine Verbiegung des quadratischen oder paralleltrapezoidischen Querschnitts der Hyalinzelle in einen gestreckt rhombischen unmöglich gemacht wird. Die untere Blatthälfte ist geschützt durch die sie bedeckenden, nächst benachbarten Blätter und somit die Aussteifung hier nicht in dem Grade erforderlich als in der oberen, frei liegenden Hälfte, oder gar der Spitze, woher die relativ sehr viel schmäleren Ringplatten fast genau parallel den Umrissen der Hyalinzellen verlaufen.

Dass die plattenförmigen Ringleisten in erster Linie die angedeutete Verschiebung oder Verbiegung der Hyalinzellen zu verhindern berufen sind, scheint mir zur Evidenz hervorzugehen aus der Construction der Platten bei Sph. rigidum, cyclophyllum und aculeatum 1). . Hier kann von Ring- oder Spiralplatten in dem bisher kennengelernten Sinne, kaum die Rede sein, denn wie die Quer-

1) Material von beiden letztgenannten Arten und $S p h$. cribrosum verdanke ich der Güte W a r n $8 \mathrm{tog}$ \&. 
schnittsansichten der Blätter genannter Arten zeigen (vergl. Fig. 58, 60-63) sind bloss den Innenwänden der Hyalinzellen breite Platten angesetzt, die sich ein Stück auf die Aussenwände fortsetzen (in Fig. 3 u. 4 bis a) um dann in eine zarte Faser von eitwa rundlich-quadratischem Querschnitt überzugehen. Der freie Rand der meniscusförmigen Platte ist beträchtlich verdickt, gleichsarn gewulstet, was sowohl in der Aufsicht des Meniscus (Fig. 60 u. 61) als im optischen Querschnitt desselben (in der Aufsicht des Blattes, Fig. 3, 4 bei a) zu erkennen ist. Wir könnten demnach hier von Ringplatten sprechen, die aus zwei breitèn, meniskenartigen Stücken und zwei schmalen, leistenartigen Stücken zusammengesetzt sind, von denen letztere an der Innenfläche der Aussenwände verlaufen.

Von nicht geringerem Interesse sind die weiteren Aussteifungsvorrichtungen bei den genannten Arten. Ein Blick auf die Blattfläche lässt zwischen den quer zur Längsaxe der Zellen verlaufenden Fasern, zahlreiche, der Längsaxe der Zelle parallel verlaufende Queranastomosen erkennen (Fig. 3, 4, 5 bei 9), die dort ansetzen, wo die meniskenförmigen Platten an den Aussenwänden der Hyalinzellen ihr Ende erreichen; von hier setzen sich häufig die Querfasern an der Oberfläche der meniskenförmigen Platte verlaufend, dieser gleichsam als Rippe aufgesetzt, bis gegen die Wand der Chlorophyllzellen fort (Fig. 61a). Somit erkennen wir hier eine sehr vollkommene, complicirte Steifungsvorrichtung, welche in erster Linie eine Formveränderung der Hyalinzellen verhüten.soll. Es wạre noch hinzuzufügen, dass gegen die Spitæe der Blätter abstehender Aeste, die Queranastomosen an Zahl sehr zunehmen ${ }^{1}$ ) und hier besonders häufig als Rippen auf den Meniskusplatten sich herabziehen. In den Blättern der herabhängenden Aeste finden wir fast nur gewöhnliche Ringplatten (Fig. 59), keine oder äusserst selten vereinzelte Queranastomosen und keine Menïscusrippen.

Einige Formen des Sph. subsecundum, zumal. contortum, zeigen die Queranastomosen ${ }^{2}$ ) und meniscusartigen Ringplatten fast in derselben Ausbildung wie Sph.. rigidum oder cyclophyllum.

In den Blättern der herabhängenden Aeste, wie herabhän g e $\mathrm{nden}$ Enden abstehender Aeste weicht der anatomische Bau nicht unbeträchtlich $a b$, insofern die Zellen zur Blattspitze hin nur wenig an Grösse abnehmen und ihre Wände nicht dicker werden; die Ring- und Schraubenplatten besitzen geringe Breite und sind von fast gleicher Bildung in der ganzen Ausdehnung des Blattes. Im Vergleich mit den Blättern abstehender Aeste tritt uns hier ein viel geringerer Aufwand von Material entgegen, weil die Ansprüche an Festigkeit viel geringere sind, und eine gleichmässige Ausbildung, weil in der ganzen Ausdehnung des Blattes die Ansprüche an Festigkeit dieselben sind.

1) Vergl. $S \mathrm{eh}$ i m per, a. a. O. Taf. XVIII, Fig. 13 u. 12.

2) Cfr. S ch im per, -a. a. O. Taf. XXЩ, Fig. 1 u. 2. 
Ueberblicken wir das bisher Mitgetheilte, so tritt uns in der Construction der Sphagnumblätter als oberstes Princip, das der Sparsamkẹit entgegen: $\mathrm{m}$ it möglichst geringen Mitteln das vorgesteckte Ziel zu erreichen; nur wo es durchaus erforderlich, finden wir einen grösseren Aufwand von Material zum Zweck der Aussteifung, und diese nach den rationellsten Principien getroffen.

Es ist mir nur ė in e Ausnahme hiervon bisher bekannt geworden, nämlich bei Sph. Mülleri Schmpr. (Sph. molle Sulliv.). Hier stimmen die Blätter der abstehenden Aeste mit den zarten Blättern der herabhängenden Aeste fast genau überein in der Construction des Zellnetzes! Vi e lle i cht ist hierin der Grund der äusserst geringen und beschränkten Verbreitung dieser merkwürdigen Art zu suchen. Die Hauptmasse der Assimilations- wie Wassersaugungsorgane, die Blätter der abstehenden Aeste, erscheinen•zu wenig geschützt gegẹn Wetterungunst etc., mithin ist diese Art ihren Genossen gegenüber benachtheiligt im Kampf ums Dasein. Meine Kenntniss dieser Art beruht leider nur auf der Untersuchung weniger getrockneter Exemplare und den Litteraturangaben, woher ich das "Vielleicht" in meiner Annahme nochmals betonen möchte.

Ziehen wir noch

\section{die Stengel- und Fruchtastblätter.}

in den Kreis unserer Betrachtung, so tritt uns auch hier im anatomischen Bau eine dem Zweck entsprechende Construction entgegen.

Im Verglëich mit den Astblättern sind die Fruchtast- und Stengelblätter von kurzer Functionsdauer und während dieser meist ganz verdeckt, letztere von den herabhängenden Aesten, erstere von den abstehenden Aesten des Schopfes, oder bei tieferer Insertion, von den Astbüscheln; daher bedarf es hier keiner besonderen Schutz- und Aussteifungstorrichtungen; in Uebereinstimmung hiemit finden wir Faserbildung in den Hyalinzellen nur selten und meist von schwacher Ausbildung.

Die Fruchtastblätter, unter allen europäischen Arten nur bei sph. rigidum Schimp. in Bezug auf anatomischen Bau den Astblättern gleich gebildet, zeichnen sich durch die Abwesenheit der Fasern in den Hyalinzellen aus wie durch die Bildung von Chlorophyll in den ihrer Lage nach den hyalinen zuzuzällenden Zellen, wie endlich dadurch, dass der Unterschied von Hyalin- und Chlorophyllzellen (zumal im unteren Theile der Blätter) auch der Lagerung nach, gänzlich aufgehoben wird und ein gleichartiges, chlorophyllführendes Gewebe das Blatt zusammensetzt. Dieses hängt offenbar mit der Function der Blätter als vorherrschend assimilatorischer Organe zusammen, deren Aufgabe in der Versorgung des Sporogoniums mit Nährstoffen besteht.

Da die Mehrzahl der die Fruchtastblätter zusammensetzenden Zellen während der ganzen Functionsperiode des Blattes lebt, so genügt der Turgor um 
die erforderliche Steifung zu bewirken. Sobald der Turgor schwindet sehen wir die Zellen mehr oder weniger bis gänzlich collabiren, wodurch die zahlreichen Verkrümmungen und Faltell der Blattfäche verursacht werden, welche wir so häufig gerade hier antreffen ${ }^{1}$ ).

In ungleich höherem Grade ạls die Fruchtastblätter, stimmen die S te n gelblätter mit den Astblättern im anatomischen Bau überein, da sie stets aus beiderlei Zellen, Chlorophyll- wie Hyalinzellen zusammengesetzt sind, welche in ihrer gegenseitigen Lagerung dieselben Verhältnisse aufweisen wie die Astblätter derselben Pflanze, nur weniger scharf ausgeprägt, doch ist ihre assimilatorische wie wasseraufsaugende F'unction meist stark reducirt; denn meist sind in den Chlorophyllzellen die Chromatophoren bald geschwunden und die Membran der Hyalinzellen, wenn sie nicht durch Fasern ausgesteift wird, entweder vollständig oder zum grössten Theil resorbirt. Wo die Membran vorhanden, wird sie entweder gar nicht, oder nur durch wenige, zarte Fasern gefestigt und entbehrt meist der, für die Astblätter so characteristischen, umwallten Poren. Die Spitze ist so wenig gefestigt, dass sie häufig fransenartig aufgelöst ist oder wie ausgefressen erscheint.

Nach Alledem müssen wir schliessen, dass die Stengelblätter in den meisten Fällen fast functionslos geworden; daher die geringe Veranlassung zur Abänderung und in Folge dessen die relativ grosse Constanz in Bezug auf Grösse, Form und anatomischen Bau, so dass wir in den Stengelblättern meist das vorzüglichste Unterscheídungsmerkmal der Arten besitzen.

Zweifelsohne sind die Stengelblätter durch Umbildung hervorgegangen aus Blättern, welche in Bezug auf Form und inneren Bau von den Astblätteru nicht oder nur wenig sich unterscheiden; das geht aus dem in anatomischer Beziehung der Hauptsache nach übereinstimmenden Bau hervor und noch mehr aus dem Umstande, dass junge Pflanzen, mögen sie aus Protonema entstehen oder aus Theilen älterer Pflanzen hervorsprossen, am Stengel stets den Astblättern gleiche oder äusserst ähnlich gebildete Blätter bilden. Ja, sind doch fast bei sämmtlichen europäischen Arten unter den zahlreichen Formen, die sich in übereinstimmender Weise innerhalb jedes Formenkreises, den wir Art nennen, wiederholen, solche beobachtet worden, die sich durch den Astblättern mehr oder weniger gleiche Stengelblätter auszeichnen: die s. g. is o phyllen. (besser hemiisophyllen) Formen. Wie mir scheint, sind diese Formen, abgesehen von einigen wenigen Fällen, durch den Standort, und zwar einen solchen, der sehr wechselnde Lebensbedingungen mit sich führt, erzeugt worden, denn die meisten isophyllen Formen habe ich an Localitäten gefunden, die zeitweilig tief unter Wasser gesetzt werden und daun vollständig austrocknen, so namentlich in der Acutifolium- und Subsecundum - Gruppe. In Folge des Austrocknens wird der

1) Cfr. meine Beiträge pag. $17-20$ und Fig. 13 u. 14. Taf. II. 
Entwickelungsgang soweit unterbrochen, dass bei wieder eintretendem Wachsthum die Sprosse . sich wie Innovationssprosse verhalten und wie diese am Stengel, wenigstens in der ersten Zeit, den Astblättern gleiche oder sehr ähnliche Blätter erzeugen. In der Cuspidatum-Gruppe wird durch permanentes Verweilen unter dem Wasser die Bildung von Fasern in den Stengelblättern begünstigt und die Gestalt derselben sehr verlängert, wodurch sie astblattähnlich werden. Wie mir scheint findet das seinen Grund darin, dass durch die Streckung des Stengels dessen Blätter in eine so güstige Lage gebracht werden, dass sie dauernd zu assimiliren vermögen und mithin den Astblättern möglichst gleich sich auszubilden streben.

In seltenen Fällen findet man an sonst normal gewachsenen Pflanzen unter normalen. Stengelblättern auch isophylle, was wohl als attavistische Erscheinung: zu deuten ist. Haben also die Stengelblätter allmälig ihre jetzige Form und Beschaftenheit erlangt, so ist.klar, dass die Arten mit faserlosen Stengelblättern, die auch stets in ihrer Form von den Astblättern weit abweichen, ihrer Entstehung nach die ältesten sein müssen. Es wären demnach in der AcutifoliumGruppe Sph. fimbriatum Wils. und Girgensohnii m. die ältesten, daneben fuscum Klingg, und subnitens $W . \& R$. (= Sph. acutifolium Ehrh. var. luridum Hüben. ampl.). Dann folgen Sph. quinquefarium (Braith.) W., Russowii W., Warnstorfii m. und tenellum Klingg. Die jüngste Art wäre Sph. acutifolium Ehrh. s. str. mit den faserreichsten, ïberhaupt im zelligen Bau wie in Bezug auf Gestalt, den Astblättern am nächsten stehenden Stengelblättern. Will man Sph. Mülleri Schimp., wie es bereits von Wà rnstorf geschehen, zu den Acutifoliis ziehen, so wäre auch diese Art als eine der jüngsten unter den Genossen aufzufassen. Da Sph. acutifolium mit beiden erstgenannten Arten wie mit Russowii und quinquefarium -unter ganz gleichen Lebensbedingungen vorkommt, so können äussere Umstände die Verschiedenheit in dem Bau der Stengelblätter nicht veranlasst haben.

Aus diesen Gesichtspunkten wäre auf die Ab- oder Anwesenheit der Fasern, im letzteren Falle, auf deren Häufigkeit und kräftigere oder zartere Ausbildung, wenn es sich um die systematische Betrachtung handelt, Gewicht zu legen, um so mehr, als mit der Faserbildung die Form der Hyalinzellen oder vielmehr die ganze Configuration des Zellnetzes Hand in Hand geht. Die faserlosen Hyalinzellen der Stengelblätter in deren oberer Hälfte sind stets fast quadratisch oder breit rhombisch, während die faserreichen gestreckt rhombisch bis schmal S-förmig gestreckt sind und dann auch häufig umwallte Poren führen, kurz den Hyalinzellen der Astblätter äusserst ähnlich sehen. Sind die Hyalinzellen rhombisch und durchgängig faserlos, so ist ihre Membran grösstentheils oder vollständig resorbirt; nur sehr selten bilden sich bei theilweiser Resorbtion zahlreiche rundliche, zart umwallte Löcher aus, die dann .gewöhnlich einander so nahe gerückt sind, dass die zwischen ihnen stehengebliebenen Membranstücke die Gestalt von 
Fasern annehmen (Fig. 16, Taf.) wofür sie auch bisher in den Beschreibungen der Autoren erklärt worden.

Dass diese falschen Fasern oder Pseudofibrillen eine von den echten Fasern oder Leisten durchaus verschiedene Bildung darstellen, ist in den Fällen besonders klar ersichtlich, wo in der. Mitte der $\mathrm{P}$ s e u d o f a s e r eine wirkliche, leistenartige Faser verläuft (Fig. 16, f), was indess bei Sph. Girgensohnii. nur sehr selten vorkommt, häufiger sich bei Sph. squarrosum und teres findet, wo die angeblichen Fasern in den Stengelblättern gleichfalls Pseudo-Fasern sind. In den normalen Stengelblättern von Sph. Girgensohnii habe ich wirkliche Faserleisten nur auf den Pseudofasern beobachtet, während bei Sph. squarrosum und teres auch ganz am Grunde der Stengelblätter wirkliche Fasern, aber nur äusserst selten, ohne Pseudofasern zur Ausbildung gelangen.

Hier möchte ich noch einer an den Stengelblättern zu beobachtenden Erscheinung gedenken, welche bisher übersehen zu sein scheint, oder die wenigstens in der Litteratur bisher keiner Erwähnung findet. Es finden sich nämlich fast durchgängig an den Stengelblättern sämmtlicher Arten, besonders auffallend in der Cuspidatum-Gruppe, an den Wünden der Hyalinzellen, am Grunde oder in der unteren Hälfte der Blätter, Falte $\mathrm{n}$, die quer, oder mehr oder weniger geneigt zur Längsaxe der Zelle verlaufen (vergl. Fig. 9, Taf. I). Bei Sph. fimbriatum und Girgensohnii, wo die Membran der Hyalinzellen fast immer resorbirt ist, können selbstverständlich diese Falten nicht vorkommen.

Oft sind diese Falten sehr scharf ausgeprägt und in grosser Zahl, dicht bei einander gestellt. 'In den meisten Fällen ist die ganze Blattfläche in der Ausdehnung der gefültelten Hyalinzellen verkrümmt oder wellig verbogen, so dass ein Causalzusammenhang zwischen dę Fältchenbildung der Hyalinzellen und der Faltenbildung des ganzen Blattes zu bestehen scheint. Sind die gefältelten Hyalinzellen septirt, so erscheint die Scheidewand zierlich wellig geschlängelt; an den Wänden der benachbarten Chlorophyllzellen dagegen ist keinerlei Fältelung: oder Verbiegung wahrzunehmen. Diese Faltenbildung vermag ich mir nur durch die Annahme zu erklären, dass bei den Hyalinzellen die Elasticität der Membran verloren geht zu einer Zeit, : wo bei den Chlorophyllzellen die elastische Membran noch durch den Turgor gespannt ist. Hört letzterer auf, so ziehen sich die Membranen der Chlorophyllzellen.zusammen, sich nicht unbeträchtlich verkürzend und die unelastische, mit den Chlorophyllzellen fest verbundene, nunmehr zu grosse Membran der Hyalinzellen, muss Falten werfen wie der unelastische, verkorkte Streifen in der radiaken Membran der Schutzscheidezellen der Gefässpflanzen, sobald nach Verlust des Turgors die Zellen sich zusammenziehen. Von einer Verkorkung oder überhaupt chemischen Veränderung der Membran lässt sich an den betreffenden Hyalinzellen der Steng̊elblätter nichts nachweisen. Die gefältelten Membranen werden ebenso wie die ungefältelten und wie die der Chlorophyllzellen durch Jod und Schwefelsäure blau gefärbt. 
In den nicht mit Fasern ausgesteiften, oder nur mit wenigen sehr zarten Fasern versehenen Hyalinzellen der oberen Stengelblatthälfte, begegnen wir fast regelmässig $\mathrm{L}$ ä $\mathrm{ng}$ s falt e n und zwar meist je einer langen Falte, die in der Richtung des längsten Durchmessers der Zelle verläuft; oft machen diese Falten den Eindruck von scharfen Rissen, welche bei Sph. squarrosum und teres thatsächlich vorhanden, durch nachträgliche Resorbtion zu weit klaffenden Spalten erweitert werden.

Endlich sei hier noch der Längsfalten erwähnt, die sich häufig an'den Membranen der Hyalinzellen in der unteren Hälfte von Astblättern bei sämmtlichen Arten finden, quer über sämmtliche Fasern einer Zelle sich erstrecken, so dass jede Faser dort, wo sie von der Falte getroffen wird, scharf eingeknickt oder verbogen erscheint. Kommen in der oberen Hälfte der Blätter Längsfalten vor, so erstrecken sie sich nur über die Membranstücke żwischen je zwei Ringplatten, weil letztere einer Knickung widerstehen, während die zarten, wenig in's Lumen vorspringenden Fasern in der unteren Blatthälfte nicht genug widerstandsfähig sind.

Zuweilen bilden sich auch tiefe Furchen in der Mittellinie der Hyalinzellen aus, namentlich bei Sph. riparium und anderen Cuspidatis (Fig. 27), aber auch in der Acutifolium-Gruppe und bei squarrosum und teres. Im Querschnitt gewinnen die Hyalinzellen dann einen etwa herzförmigen Umriss.

Wir haben bisher die Hyalinzellen in Bezug auf ihre Aussteifungsvorrichtungen kennen gelernt, wenden wir uns jetzt der Betrachtung jener Einrichtungen zu, welche dieselben Zellen befähigen Wasser rasch aufzunehmen und festzuhalten, der

\section{Befrachtung der Poren.}

Sind die Hyalinzellen in F'olge ihres engen Lumens befähigt bedeutende Capillarkräfte zu entwickeln, so können diese doch erst wirksam werden durch die Perforation der Wände, insofern hierdurch nicht nur ein rascher und leichter Eintritt des. Wassers, sondern auch ein Entweichen der eingeschlossenen Luft ermöglicht wird. Wir sehen daher bei allen Torfmoosen mit Ausnahme des auch sonst abweichenden und eigenthümlichen Sph. Pylaiei die Hyalinzellen der Astblätter mit zahlreichen, grösseren oder kleineren Poren versehen. Bei vielen Arten sind die Rindenzellen des. Stengels gleichfalls perforirt, wenn auch nur durch ein Loch, wie bei den Acutifoliis, oder die Rindenzellen der Aeste, die merkwürdigen Retortenzellen. Bei Sph. Pylaiei habe ich nur hie und da zarte, durch Resorbtion entstandene, nicht umwallte Löcher gefunden.

Mit Ausnahme der Cuspidata, welche von allen Torfmoosen am meisten an reichliches Wasser gebunden sind und oft ganz untergetaucht wachsen, sind die Poren, wenigstens an der Aussenseite der Blätter, zumal an deren freier Spitze 
von einer Ringfaser umgeben, d. h. umwallt. Bei den Cuspidatis fehlen zwar die Porenringe nicht ganz, doch finden sie sich im Ganzen selten.

Der Zweck der „Ringfaser" als eines Schutzmittels gegen Einreissen der Löcher und mithin einer nachtheiligen Vergrösserung der Oeffnungen in der Zellhaut, ist evident, da bei allen den während der längsten Dauer der Vegetationszeit, wenigstens in ihren oberen Theilen von Luft und nicht von Wasser umspülten Arten, die Löcher umwallt sind, zumal an den freien Theilen der Blattoberfläche. Sehen wir uns aber die bisher nicht erkannte, eigenthümliche Form der ringförmigen Einfassung näher an, so drắngt sich uns die Annahme auf, dass noch ein anderer Ziweck mit der Umwallung verbunden sein könnte.

Nach den Beschreibungen zu urtheilen, hat man bisher wohl allgemein die den Porus umgebende Ringfaser, gleich den Ring- und Spiralleisten, ·für eine nur wenig in's Lumen der Hyalinzelle hineinragende Leiste oder Faser von ringförmiger Gestalt gehalten. Sehen wir uns darauf die Abbildungen bei Schimper an, so sollte man meinen, dass die Ringfaser kaum merklich in's Lumen vorspringt oder durch eine sanfte Einbiegung des Lochrandes nach Innen zu Stande kommt (vergl. Schimper a. a. 0. Taf. VI, Fig. 7 und Taf. XXVII). Vergleicht man mit diesen Abbildungen die auf den hier beigegebenen Tafeln. dargestellten Blattquerschnitte (Fig. 10, 11, 12, 31, 48, 52, 55-60, 62), so werden an diesen, ebenso wie die in's Lumen der Hyalinzellen weit hineinragenden Ringplatten, sogleich die merkwürdigen, trichterartigen, nach Aussen ebenso wie nach Innen zart gewulsteten, oder richtiger, von einem häutigen Doppelsaum umgebenen Löcher in die Augen springen. Besonders scharf ausgeprägt tritt die eigenthümliche Bildung an den auffallend kleinen Löchern in der Spitze der Blätter abstehender Aeste von Sph. Warnstorfii uns entgegen (Fig. 10), auch bei $S p h$. fustum und den übrigen Acutifoliis wie auch bei den Subsecundis und etwas modificirt bei den Cymbifoliis und bei rigidum. Die Form des Porendurchschnitts erinnert in sehr auffallender Weise an die Gestalt des Spaltöffnungsdurchschnitts zahlreicher Phanerogamen, wo die Spalte zwischen den Schliesszèllen einen s. g. Vor- und Hinterhof bildet. Die Wandung des Loches verläuft nicht glatt, sondern zeigt zwei Einbuchtungen, die durch einen mittleren Vorsprung getrennt werden, oder mit anderen Worten: die Lochwandung wird von drei in's Lumen (des Loches) vorspringende Ringe eingeengt, zwischen denen sich zwei ringartige Erweiterungen (oder Einbuchtungen in die Lochwand) befinden. Bald sind alle drei Ringe gleich stark entwickelt, bald der mittlere am stärksten, bald die beiden äusseren oder auch nur einer von beiden und zwar gewöhnlich der nach aussen liegende.

Entsprechend der Breitenabnahme der Ringplatten von der Spitze zur Basis des Blattes, sehen wir auch die.Umwallung der Löcher an Mächtigkeit abnehmen, umgekehrt dagegen den Durchmesser der Löcher von der Spitze zur Basis des Blattes zunehmen, ebenso wie die Grösse der Zellen; doch nehmen im Allge- 
meinen die Löcher von der Basis zur Spitze des Blattes hin nicht in dem Verhältniss an Grösse ab als die Zellen, so dass die kleineren Zellen der Spitze durch relativ grössere Löcher vor den Zellen der unteren Blatthälfte sich auszeichnen. Um so auffallender treten daher bei Sph. Warnstorfii $m$. und einigen Formen des Sph. fuscum wie bei Sph. Wulfianum die Löcher in der oberen Blatthälfte durch ihre ạusserordentliche Kleinheit hervor. Die Löcher der letztgenannten Art sind durch die äusserst stark nach innen vorspringende Umwallung ausgezeichnet (Fig. 42, Taf. IV) während der äussere Rand nur wenig oder kaum merklich vorspringt.

Wie die Aussteifungs-Ringe und Spiralen der Hyalinzellen aus Sparsamkeitsrücksichten in der unteren Blatthälfte, die hinlänglich geschützt ist, nur als zarte Fasern zur Entwickelung gelangen, so unterbleibt die Umwallung der Poren häufig an Orten, wo eine Festigung der Lochründer entbehrlich ist, an der Innenfläche der Blätter und auch an der Aussenftäche, wenn diese nach Innen eingeschlagen ist, so namentlich bei den Acutifoliis und Squarrosis. Bei den Cuspidatis, wie bereits erwähnt, ist die Umwallung der Löcher sehr selten ausgebildet, da sie hier durch das die Pflanzen umgebende Medium überflüssig wird; ebenso verhält sich Sph. macrophyllum Bernh.

Nicht ohne Interesse ist bei den Cuspidatis eine nähere Untersuchung der Löcher in Bezug auf ihre Vertheilung, Häufigkeit und Grösse. Bei den untergetauchten oder schwimmenden Formen, (formae immersae S chimpers), sind die Löcher meist sehr klein und sparsam an den Blättern der abstehenden wie herabhängenden Aeste (wenn letztere überhaupt vorhanden) in jeder Beziehung gleich gebildet. Bei den aus dem Wasser hervorragenden Formen (formae emersae $\mathrm{Schimpers)} \mathrm{sind} \mathrm{die} \mathrm{Löcher} \mathrm{in} \mathrm{den} \mathrm{Blättern} \mathrm{abstehender} \mathrm{Aeste} \mathrm{meist} \mathrm{klein}$ und ziemlich zahlreich, in den Blättern der herabhängenden Aeste meist gross bis sehr gross und zumal in der oberen Blatthälfte sehr zahlreich. Als Typus dieser Ausbildung kann Sph. riparium genannt werden. Zwischen diesen beiden angeführten Extremen kommen alle denkbaren Zwischenbildungen vor, je nach dem Standort. Als besonders characteristisch für die ganze Gruppe ist die Ausbildung von Löchern in den der Blattspitze zugekehrten Spitzen der Hyalinzellen, entweder nur an der Aussenseite des Blattes (einseitige Spitzenlöcher) oder auch an der Innenseite (zweiseitige Spitzenlöcher). Diese Spitzenlöcher treten meist nur in der oberen Hälfte des Blattes auf und zwar sind sie beim RipariumTypus in den Blättern der herabhängenden Aeste zweiseitig, sehr gross und zahlreich '), in den Blättern der abstehenden Aeste auch meist zweiseitig, aber viel kleiner und weniger zahlreich. Ausserdem sind grosse zweiseitige Spitzenlöcher bei riparium und einigen anderen Formen an den Flanken der Blätter abstehender Aeste eine auffallende Erscheinung. 
Hiezu möchte ich bemerken, dass die erwähnten Verhältnisse bei den Cuspidatis nur mit Hilfe tingirender Mittel zu erkennen sind wegen der aùsserordentlichen Durehsichtigkeit der Zellhaut. Die etwas umständliehe und nicht immer gleichförmig ansfallende Tinction vermittelst Jod und Schwefelsäure, kann in einfachster: und bequemster Weise durch Anwendung von Anilinfarbstoffen ersetzt werden; unter welchen ich Methylviolett als besonders geeignet gefunden habe. Man tauche feuchte Aeste in eine dunkle Lösung des genannten Farbstoffes nur auf ein Paar Minuten, wasche dann aus und löse in üblicher Weise die Blätter ab um sie in einem Tropfen Wasser auf dem Objectträger auszubreiten. Dagegen ist die Herstellung gefärbter Dauerpräparate eine recht umständliche, denn in Glycerin oder Gelatineglycerin wird der Anilinfarbstoff aus der Membran der Sphagnumblätter vollständig extrahirt. Die Fixirung der Tinetion gelingt nur in Balsam ${ }^{1}$ ).

Unter allen untersuchten Arten steht Sph. rigidum Schmpr. einzig da in Bezug auf Porenbildung, insofern hier die Löcher nicht nur an den Aussenwänden auftreten, sondern auch in den zweien Hyalinzellen gemeinsamen Wänden grosse umwallte Löcher gebildet werden, so dass die Hyalinzellen unter einander in offene Communication treten. (Fig. 58, L). Es kommt diese eigenthümliche Bildung dadurch zu Stande, dass die Membranen der Hyalinzellen sich äusserst stark vorwölbend, an den Berührungsflächen mit einander verwachsen und dort, wo zwei Löcher (die hier dicht an den Commissuren mit den Chlorophyllzellen stehen) auteinander treffen, diese zu einem Loch verschmelzen. Diese inneren Löcher bilden sich sowohl an den langen als kurzen Wänden der Hyalinzellen aus.

Ergiebt sich aus der bisherigen Betrachtung der Löcher zur Evidenz der Zweck der Umwallung als einer festigenden Einrichtung, so ist uns die Bedeu-

1) Da ein tingirtes Sphagnumblatt zu den schönsten und zierlichsten mikroscopischen Objecten gehört, welche uns die Pflanzenwelt liefert, so mag das Verfahren tingirte Dauerpräparate herzustellen, hier mitgetheilt werden.

Man bringe, um Ueberfärbung zu vermeiden, die betreffenden Sphagnum-Aeste zunächst in eine diluirtc wässrige- Anilinlösung (Methỳlviolett, Fuchsin, Bismarkbraun, Nacbtgrün etc. nur Anilinblau eignet sich nicht) und lasse die Aeste hier einige Tage liegen, bis sich die Lösung verfärbt; es wird ihr der Farbstoff von den Blättern entzogen. Darauf breitet man die Blätter auf einem Objectträger aus, bedeckt mit einem Deckglass und fügt am Rande des letzteren diluirten Alcohol zu, den man von der entgegengesetzten Seite des Deckglases mit Fliespapier abzieht und ersetzt allmälig den diluirten Alkohol. durch immer stärkeren bis zu $96 \%$. Tbut man die Aeste gleich in starken Alcohol, so schrumpfen die Blätter zusammen; auch beim Uebertragen aus schwächerem Alcohol in stärkeren sind beträchtliche Verkrümmungen des Blattes nicht zu vermeiden, liegt aber das Deckglas den Blättern dicht auf, so behalten sie bei allmäliger Wasserentziehung ihre Gestalt. Die so entwässerten Blätter werden in Bergamottöl gebracbt und nach einigem Verweilen in diesem in Canadabalsam gebettet. Auf einer dünn ausgestrichenen Schicht des Balsams lassen sich die Blätter auf dem Objectträger nach Belieben aufstellen und ordnen; man bestreiche nun die aufzulegende Fläche des Deckglases mit flüssigem Balsam und legt dasselbe auf die Blätter. Das Deckglas muss beschwert werden mit einem geeigneten Gewicht, das bis zum Eintrocknen des Balsams liegen bleibt. Ohne diese Belastung wird das Deckglas von den gewölbten Blättern bald emporgehoben, Luft tritt ein und das Präparat wird. unbrauchbar. 
tung der merkwürdigen Form der Umwallung noch nicht klar geworden. Da Resorption der Membran ohne Umwallung der resorbirten Stelle zu Stande kommt, so ist die eigenthümliche Form der Umwallung unabhängig von dem Resorbtionsvorgange. Käme es nur darauf an, den Lochrand gegen Einreissen zu schützen, so wäre ein einfacher Ringwulst nicht nur ausreichend, sondern vielleicht wirksamer als der complicirt gebaute, nach Aussen wie Innen oft sehr fein zugeschärfte Lochrand. Es lässt sich denken, dass beim Eintrocknen etwa die beiden Ränder sich einander näherten, wodurch das Lumen des Loches verkleinert würde; doch wäre diese Verengerung des Loches in den meisten Fällen eine so geringe, dass sie nicht in Betracht käme. Somit erscheint es unthunlich den umwallten Löchern die Rolle von Oeffnungen mit wechselnder Weite des Lumens zu vindiciren, wie wir solche in den Spaltöffnungen der höheren Gewächse kennen, auch ist. es mir nicht geglückt eine Verschiedenheit in der Stellung der Lochränder im feuchten und trocknen Zustande wahrzunehmen. Bedeutungslos aber können die Doppelränder der Poren nicht sein, sonst hätten sie sich nicht gebildet und e rhalten. Die wiederholt von mir beobachtete Thatsache, dass die Arten, bei welchen die Löcher einen stark entwickelten Doppelrand zeigen wie bei Sph. Warnstorfi, gegen Austrocknen besser geschützt sind als die Arten bei denen die Umwallnng der Poren gering ist oder gar ganz fehlt, drängt zur Annahme, dass die eigenthümliche Umwallung der Löcher eine Vorrichtung darstellt, vermittelst welcher das in den Zellen befindliche Wasser leichter zurückgehalten oder Wasser. von aussen, etwa in Form von Thau oder Nebel, leichter aufgenommen werden kann. Jedenfalls ist es eine auffallende, oft von mir beobachtete Erscheinung, dass bei dicht neben und durcheinander wachsenden Rasen von Arten aus der Acutifolium-Gruppe und Cuspidatum-Gruppe, letztere zu allererst trocken werden, zuletzt Sph. acutifolium seine Feuchtigkeit verliert, das unter allen Gruppengenossen (gleiche Bedingungen vorausgesetzt) am längsten seine Feuchtigkeit bewahrt. Eine bessere'Begründung, oder aber auch Widerlegung dieser Annahme von der Bedeutung der doppelrandigen Lochumwallungen bleibe der Zukunft vorbehalten.

Wir können diesen Abschnitt, welcher sich mit den Einrichtungen der Wasseraufsaugung beschäftigt nicht schiliessen, ohne nochmals der Steifungsvorrichtungen zu gedenken und deren Bedeutung auch in Hinsicht der Wasser-Aufnahme und Abgabe zu·beleuchten.

Selbstverständlich wird ein Capillarröhrchen (was ja die Hyalinzelle eines Sphagnumblattes ist) um so ergiebiger Wasser anziehen und dieses festhalten, je grösser die Oberfläche des Lumens bei gleiehem Durchmesser des letzteren ist, also je mehr Rauhigkeiten die Wand. des Capillarrohrs bietet. Offenbar wird nun durch die tief in's Lumen der Hyalinzellen hineinragenden Ring- und Spiralplatten die innere Oberfläche der betreffenden Zellen ganz ausserordentlich vergrössert und somit die Adhäsionskraft der Zellen für Wasser sehr gesteigert. Wir sehen, 
dass in der unteren Hälfte der Blätter -abstehender Aeste die Steifungsringe nụr wenig in's Lumen vorspringen, weil dieser Theil durch Deckung von Seiten benachbarter Blätter geschützt ist gegen Verletzungen; er ist aber auch ebenso geschützt gegen V e r d u n s t u n g, und somit kann aus diesem Gesichtspunkt hier die innere Oberflächenvergrösserung eine geringere sein. Wenn wir dagegen die Papillen an den Wänden der Hyalinzellen bei Sph. papillosum Ldbg., squarrosum, teres und Wulfianum und die Kammfasern bei Sph. Austini Sulliv, welche Gebilde jedenfalls neben einer anderen, später zu besprechenden Hauptaufgabe noch als Nebenzweck den der Oberflächenvergrösserung besitzen, meist in der unteren Blațthälfte stärker und zahlreicher ausgebildet finden als in der oberen, so wird dieser Mangel durch die stärkere Entwickelung der Ringplatten in der oberen Blatthälfte reichlich compensirt.

Ueberblicken wir nun noch einmal kurz den Bau der Hyalinzellen, indem wir den durch dieselben angestrebten Zweck in's Auge fassen und die je nach der ortlichen Lage ungleichen Anforderungen in Bezug auf Festigkeit und Wasserabsorption berücksichtigen, so müssen wir bekennen, dass uns überall eine ho chst vollkommene Construction entgegentritt, die mit einem möglichst geringen Aufwande von Mittelnerreichtwird.

Fassen wir jetzt die den assimilatorischen Zwecken dienenden Elemente in's Auge:

\section{die Chlorophyllzellen.}

Da die Blätter der abstehenden und herabhängenden Aeste dem Lichte in sehr ungleichem Maasse exponirt sind, so können wir erwarten in Bezug auf die Lagerung und Form der Chlorophyllzellen in den beiderlei Blättern eine Verschiedenheit anzutreffen, was thatsächlieh der F'all ist ; wir wollen daher zunächst von den Blättern der abstehenden Aeste sprechen und an diesen auch nur zunächst die freie, unbedeckte obere Hälfte in's Auge fassen.

Bei der Dünne und Durchsichtigkeit der Sphagnumblätter sollte man meinen, dass es gleichgültig sei wie die Chlorophyllzellen liegen, Licht müssen sie ja in jedem Fall in genügender Menge erhalten. Wie mir scheint, muss man bei der Beurtheilung der verschiedenen Lagerung der Chlorophyllzellen in den Sphagnumblättern nicht von dem Gesichtspunkt des Lichtbed ürfn isses, sondern des Lichts chutzes ausgehen.

Bekanntlich ist das Chlorophyll der Muscineen meist lichtscheu, um es kurz auszudrücken, im Vergleich zum Chlorophylle der meisten höheren Gewächse, das, wie wir wissen, ebenfalls vor zu vielem Licht geschützt werden muss um nicht zerstört zu werden. Wir finden daher die Mehrzahl der Laub- wie namentlich Lebermoose an mehr oder weniger schattenreichen Orten, ja im tiefsten Waldesdunkel gedeihen und wissen, dass bei diesen Gewächsen das die Chlorophyllkörner einschliessende Protoplasma in Folge Lichtreizes Bewegungen ausführt, 
durch welche Chlorophyllkörner aus der Schattenlage in die Lichtlage versetzt werden, d. h. in eine Stellung gebracht werden, bei der sie viel weniger Licht empfangen als vorher.

Unter der Voraussetzung nun, dass das Chlorophyll der Torfmoose ebenso wie das der übrigen Muscineen lichtempfindlich ist und nicht ungeschützt der Sonne exponirt werden darf, werden uns die Form- und Lagerungsverhältnisse der Chlorophyllzellen wie die bei den Torfmoosen so sehr verbreiteten, starken Pigmentir u n g en der Zellwände verständlich.

Gehen wir von der ursprünglichen Lagerung der Chlorophyllzellen im jugendlichen, noch in Entwickelung begriffenen Blatte aus. Ein Querschnitt zeigt uns hier die beiderlei Zellen an der Aussen- wie Innenfläche des Blattes plan und von gleicher Ausdehnung in der Richtung senkrecht zur Blattfläche. Die Hyalinzellen erscheinen etwa quadratisch, die Chlorophyllzellen rectangulär, um ein Drittel bis zur Hälfte schmäler als erstere. Bei der weiteren Entwickelung dehnen sich die Hyalinzellen beträchtlich aus, ihre Aussenwände mehr oder weniger abrundend und über die Chlorophyllzellen vorwölbend, derart, dass letztere entweder (meist) an die Innenfläche oder (selten) an die Aussenfläche des Blattes gedrängt werden oder auch beiderseits gleichmässig umschlossen werden. In beiden Fällen ändert sich die ursprüngliche rectanguläre Gestalt der Chlorophyllzellen nicht unbeträchtlich; im ersten Fall wird sie dreieckig, indem die eine Aussenwand gebrochen und soweit ausgezogen wird, bis aus dem Rechteck ein Dreieck geworden, dessen Spitze entweder nach der Aussenfläche (Fig. 18, 19, 21) oder nach der Innenfläche des Blattes zu liegen kommt (Fig. 26, 27, 28); im zweiten Fall werden beide Aussenwände gebrochen und soweit ausgedehnt, bis der Querschnitt linsenförmig gewoṛden (Fig. 42, 43, 48, 51, 56-63). In einigen Fällen behalten aber die Chlorophyllzellen ihren ursprünglichen rectangulären Querschnitt fast genau bei, nur die mit den Hyalinzellen verwachsenen Wände ein wenig in letztere hineinwölbend, wie bei.einigen Formen der Subsecunda (Fig. 31, 39) oder die Chlorophyllzellen bleiben rectangulär oder werden fast quadratisch oder paralleltrapezoidisch und trennen die Hyalinzellen, welche ein nahezu kreisrundes Lumen gewonnen, vollständig von einander, wie das gewöhnlich bei den Blättern der herabhängenden Aeste in den Gruppen der Acutifolia, Cuspidata"und Squarrosa der Fall ist (Fig. 24, 13, 14, 15, 46).

In der Jugend, wo das Chlorophyll am leichtesten durch Lichteinwirkung zersetzbar ist, wird es sehr wirksam geschützt durch die Einbettung der jungen Sprosse in den dichten Astschopf.

Durch die Stellung, wie sie uns bei den Acutifoliis und zum Theil bei den Cymbifoliis entgegentritt; $d$. h. wo die dreieckigen Chlorophyllzellen an die Innenfläche gerückt sind und von den Hyalinzellen nach der Aussenfläche des'Blattes hin vollständig überwölbt werden, ist das Chlorophyll direct einfallenden Lichtstrahlen entzogen. Noch wirksamer gegen directes Licht erscheint mir die all- 
seitige Umschliessung der Chlorophyllzellen, bei linsenförmigem Querschnitt letzterer, wodurch die Chloroplastiden nur unter sehr spitzen Winkeln von den Sonnenstrahlen getroffen werden können und mithin die Lichtwirkung bedeutend abgeschwächt wird.' Doch in beiden Fällen scheint dieser Schutz nicht zu genügen, wenn die Pflanzen an der Sonne sehr exponirten Localitäten wachsen. Dann sehen wir Farbstoffe auftreten, welche die Zellhaut sehr intensiv tingiren, wodurch offenbar ein ,L i c h t s c h i m meschaffen wird.

So ist die auf sonnigen Hochmooren verbreitetste Art Sph. fuscum Klingg. wie der Name sagt, tief braun gefärbt; Formen derselben Art welche an beschatteten Stellen wachsen sind lichtbraun bis hellgrün-bräunlich gefärbt. Die an sonnigen Stellen des Hochmoors ebenfalls sehr verbreiteten Formen des Sph. tenellum Klingg. v. rubellum (= Sph. rubellum Wils.) sind tief roth gefärbt, desgleichen die hier wachsenden F'ormen des Sph. cymbifolium Ehrh. (Sph. medium Limpr.), während andere dieser Art angehörende Formen wie das nah verwandte Sph. Austini Sulliv. braun bis tief braun gefärbt sind. Die rothe Farbe ist ferner bei den Auctifoliis, mit Ausnahme des schattenliebenden Sph. fimbriatum Wils. und sph: Girgensohnii m., sehr verbreitet, ja fast stets vorhanden, zumal bei den an sonnigen Plätzen wachsenden Formen. Bei Sph. Girgensohnii $m$. tritt, wenn es an freien, lichten Plätzen wächst, ein gelb-brauner bis brauner Farbstoff auf.

Eine ganz exquisite Lichtschutz-Vorrichtung tritt uns, wie mir scheint, in den Papillen des Sph. papillosum Lindbg., Sph. squarrosum, teres und Wulfianum, wie in den Kammfasern des Sph. Austini Sulliv. entgegen. Bedeutungslos können die genannten Gebilde für die betreffenden Pflanzen nicht sein; die Adhäsion für Wasser wird zwar durch dieselben vermehrt, aber nur in beschränktem Maasse, da sie sich nicht in der ganzen Ausdehnung der Wände der Hyalinzellen finden, sondern nur soweit, als letztere mit den Chlorophyllzellen verwachsen sind und das ist es eben, was uns die Ansicht aufdrängt, dass wir es hịer mit einer Einrichtung zu thun haben, welche in erster Linie den Chlorophyllzellen zu Gute kommt; einen anderen Nutzen aber, als den eines , L i c h t schirms", könnell die Chlorophyllzellen wohl kaum aus dieser Einrichtung ziehen.

In der Subsecundum-Gruppe, wo die im Querschnitt tonnenförmigen oder linsenförmigen Chlorophyllzellen gerade in der Mittè-zwischen den Hyalinzellen stehen und dem Licht von beiden Seiten gleich freien Zugang in's Innere gestattet ist, wird durch die sehr dicken Aussenwände (Fig. 31), zumal an der Aussenseite des Blattes, die Wirkung des Lichtes einigermaassen abgeschwächt, mehr aber jedenfalls durch die in dieser Gruppe häufig auftretenden dunkelgelben bis* schwarzbraunen Pigmente, welche nie bei den an sonnigen Orten wachsenden Individuen fehlen.

Am meisten dem Lichte exponirt ist das Chlophyll in der Cuspidatum- 
Gruppe, da die freien Wände der Chlorophyllzellen an der Blattaussenfläche liegen; vielleicht wird die Intensität des. Lichtes gemildert durch die d a c ha r tig e Zuspitzung, resp. Einsenkung der freien. Wand (Fig. 22, 25, 28), eine Erscheinung, die ich fạst durchgängig in dieser Gruppe, freilich nicht in der ganzen Ausdehnung der Blätter gefunden habe und darin liegt, wir ich zu zeigen hoffe, die wahre Bedeutung dieser Erscheinung. In Folge der dachartig zugespitzten, resp. eingesenkten Aussenwände kann das Licht nur gebrochen in's Innere der Zellen gelangen; das käme aber nur den betreffenden Zellen zu gute. Die eigentliche Bedeutung dieser Erscheinung scheint mir aber darin zu bestehen, dass beim Verlust des Turgors (die Cuspidata verlieren ausserordentlich leicht das imbibirte Wasser) die Chlorophyllzellen sich in der Weise zusammenziehen, dass der Winkel der dachartigen Zuspitzung kleiner wird, oder was dasselbe, die beiden inneren Wände der Chlorophyllzelle sich einander nähern; . dadurch wird die Blattfläche in der Ausdehnung jener, die dachartige Zuspitzung besitzender Zellen, sich zusammenziehen und da andere Theile der Blattfläche solch' eine Contraction nicht erfahren,. nämlich überall da, wo die besagte Erscheinung an der Aussenwand der Chlorophyllzellen nicht vorkommt, so wird die Blattfläche sich wellig verbiegen oder kräuseln, was thatsächlich der Fall und ein auszeichnendes Merkmal der Cuspidata bildet. Anders vermag ich mir die so characteristische Kräuselung der eintrocknenden Cuspidatum-Blätter nicht zu erklären und in der Kräuselung vermag ich wiederum nur ein Schutzmittel gegen allzu starke Insolation zu erblicken. Durch braune Farbstoffe sind nur wenige Formen geschützt, wie Sph. Lindbergï Schmpr., das der Kräuselung ermangelt und dessen Chlorophyllzellen die dachartige Zuspitzung nicht zeigen (Fig. 30). Da im turgescirenden Zustande die meisten Formen der Cuspidata gegen Licht weniger als die übrigen geschützt sind, so erklärt sich die Thatsache, dass, mit Ausnahme der im Waldschatten wachsenden Formen, welche oft tief grün sind, wie oft bei $S p h$. riparium und recurvum der Fall, die meisten Cuspidata sich durch bleiches, weissliches Ansehen vor allen Gattungsgenossen auszeichnen.

Bei den Squarrosa (oder Papillosa) sind die Chlorophyllzellen zwar auch meist dreieckig (im Querschnitt) und wie bei den Cuspidatis von der Aussenseite her zwischen die Hyalinzellen gekeilt, doch, wie ein Blick auf die Figg. 36-47 zeigt, sind die schmal dreieckigen Lumina in die Mitte zwischen den Hyalinzellen gerückt, indem die Aussenwände der Chlorophyllzellen stark verdickt sind, oder die Zellen sind (gerade in der freien Spitze des Blattes) linsenförmig, allseitig umschlossen und dann sind die Chlorophyllzellen hier durch P a pill en geschützt. Ausserdem sind es zum grössten Theil Waldbewohner und die an sonnigen Orten vorkommenden Formen, zumal von teres, sind meist dunkelbraun gefärbt.

Bei Sph. rigidum Schmpr., Angstroemii Hartm., aculeatum Warnst., cyc- 
lophyllum S. $L$. und zahlreichen Formen des Sph. palustre $L$., so Sph. medium Limpr. und papillosum Lindbg., sind die Chlorophyllzellen durch die sie umschliessenden Hyalinzellen wie durch braune und rothe Farbstoffe geschützt.

Wir haben bisher nur die freie, unbedeckte obere Hälfte der Blätter berücksichtigt. Betrachten wir nun die Lagerungs- und Formverhältnisse der Chlorophyllzellen auch in der unteren bedeckten Blatthälfte, die natürlich weniger Licht empfängt als die obere Hälfte. Die längst bekannte Thatsache, dass in der Acutifolium-Gruppe z. B. in der unteren Hälfte der Blätter die Hyalinzellen sich nicht (wie in der oberen Hälfte) über die Chlorophyllzellen vorwölben, so dass letztere an ihrer Aussenfläche ungedeckt sind, wird uns nunmehr verständlich; es wird dem gedämpften Licht freier Zugiang gewährt ins Innere der Chlorophyllzellen; eine noch weitere Abschwä́chung des Lichts durch Vorwölbung der Hyalinzellen würde den Assimilationsvorgang beeinträchtigen. (Man vergleiche Fig. 10, 11, 12, Querschnitte aus der oberen Blattthälfte und Fig: 13, 14, 15, Querschnitte aus der unteren Blatthälfte, mit einander). Ebenso, oder noch eclatanter ist es bei Sph. squarrosum, teres. und Wulfianum (Fig. 36-47). Bei den Arten, wo die Chlorophyllzellen von den Hyalinzellen in der oberen Blatthälfte umschlossen werden, finden wir in der unteren Blatthälfte, bis auf Sph. rigidum und aculeatum, die Chlorophyllzellen mehr oder weniger dreieckiges Lumen gewinnen und bis an die Innenfläche des Blattes heranrücken.

Dass Form und Lagerung der Chlorophyllzellen abhängig sind von dem Maass des die betreffenden Blätter treffenden Lichts, tritt uns am deutlichsten entgegen an den Blättern der herabhängen Aeste. Wie schon oben bemerkt wurde, ist hier der Querschnitt der Chlorophyllzellen rectangulär bis quadratisch oder trapezoidisch, wodurch die Hyalinzellen vollkommen von einander getrennt werden und somit die ersteren das bereits mehrfach gebrochene, 'reflectirte und gedämpfte Licht, ohne weitere Abschwächung durch Vorwölbung der Hyalinzellen, empfangen; eine weitere Abschwächung des Lichts wäre hier eher nachtheilig als vortheilhaft.

Ein Umstand aber ist hierbei noch zu berücksichtigen, nämlich dass die Chlorophyllzellen in den Blättern der herabhängenden Aeste in der Mitte zwischen den Hyalinzellen stehen, auch bei den $\mathrm{Cuspid}$ at is (Taf. III, 24), obwohl hier der Beleuchtungsverhältnisse wegen solch' eine Lagenveränderung nicht nothwendig erscheint. Somit ist wohl mit der centralen Lage der Chlorophyllzellen hier noch ein anderer Zweck verbunden. Da den herabhängenden Aesten wohl in erster Linie die Saugung des Wassers von unten her obliegt, so könnte die erwähnte Stellung der Chlorophyllzellen für die Wasserbewegung von unten nach oben vortheilhaft sein, insofern als dadurch zwischen den aufeinanderliegenden Blättern relativ weite capillare Gänge oder Röhren hergestellt werden, die einer ergiebigen Verbreitung des Wassers günstig sind. Vielleicht hängt aber auch die centrale Stellung der Chlorophyllzellen mit der Zugfestigkeit zusammen, 
insofern durch diese Stellung die von den Chlorophyllzellen hauptsächlich zu tragende Masse des wassererfüllten Blattes, gleichmàs ig zu beiden Seiten der Träger vertheilt wird. Diese letzte Annahme erscheint mir am plausibelsten.

Es erübrigt nun noch darauf hinzuweisen, dass in Bezug auf Festigkeit in der Construction des ganzen Blattes, die Form und Lagerung der Chlorophyllzellen von wesentlichem Einfluss ist.

Der Antheil, den die Chlorophyllzellen an der Gesammtfestigkeit des Blattes haben, schëint mir sehr verschieden, ja nachdem dieselben von den Hyalinzellen gänzlich umschlossen werden, oder letztere mehr oder weniger von einander trennen. Im ersten Fall, wie bei Sph. rigidum (Fig. 58, 60, 61) Sph. aculeatum (Fig. 63) und medium sind die Wände der Chlorophyllzellen relativ sehr dünn und sie können daher nur wenig zur Festigkeit des Ganzen beitragen. Dafür sind hier die Berührungsflächen der Hyalinzellen unter einander sehr gross und deren Aussteifungsvorrichtungen, (wenigstens bei beiden erstgenannten) sehr vollkommen und eigenthümlich, so dass der Hauptantheil in Bezug auf Steifigkeit des Blattes den Hyalinzellen zukommt.

Im zweiten Fall finden wir die Wände der Chlorophyllzellen meist beträchtlich rerdickt, zumal an den freien Flächen, wodurch nicht selten ihr Lumen in die Mitte zwischen Aussendie Aehnlichkeit mit denen des ersten Typus eine sehr grosse wird, so dass in Folge dessen von verschiedenen Autoren die Form und Lagerung der Chlorophyllzellen bei derselben Art sehr verschieden angegeben wird ${ }^{1}$ ).

1) So schreibt $\mathrm{S}$ ch imper Sph. squarrosum (incl. teres), ,cellulae virides compressae un dique i n cl u s a e $e^{r \epsilon}$, während $\mathrm{L}$ in $d$ ber $\mathrm{g}^{1}$ ) den Sachverhalt richtig angiebt indem er sagt : ,cellulae chlorophylliferae subrectangulari - ovatae, in margine convexo positae ibidemque distincte rotundatae, in marginibus utrinque perfecte liberae nec ab eisdem inanibus inclusae, praesertim ad marginem convexum sat valde incrassatae, lnmine subelliptico et majusculo“; ebenso Limp rich t ${ }^{2}$ ): „Chlorophyllzellen der Astblātter bald triangulār und der Aussenfläche genāhert, doch meist in der Mitte zwischen den Hyalinen und beiderseits frei; im oberen Blattheile beiderlei Zellen von gleicher Höhe, daher die hyalinen Zellen biplan,

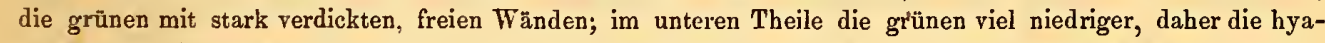
linen biconves". Dagegen weichen die beiden letztgenannten Autoren in ihren Angaben betreffs der Chlorophyllzellen von Sph. Angstroemii nicht unbeträchtlich von einander ab. L i n d b e r g ${ }^{3}$ ) sagt zutreffend: „Cellulae chlorophylliferae subovatulo - oblongae, fere in medio, licet marginem concavum distinctiuscule propius, inter easdem inanes positae, sed ab is $\mathrm{n}$ on in clusae, ut in marginibus, praesertim in eodem concavo, liberae, parum et conformiter incrassatae, lumine oblongo et magno." Dagegen L i mprich t ${ }^{4}$ ) "grüne Zellen im Querschnitt schmal elliptisch, centrirt und $\mathrm{r}$ ings von den biplanen hyalinen eingeschlossen, unten beiderseits frei und die hyalinen biconvex.

. Noch grösser finden wir die Differenzen in den Angaben der Lagerungs- und Formverhältnisse der Chlorophyllzellen bei verschiedenen Autoren in Bezug auf Sph. papillosum Ldbg. die sich aus der thatsächlichen groșsen Variabilitāt der beregten Verhältnisse erklären.

1) Europas och Nord-Amerikas Hritmossor. 1882, Helsingfors, pag. 42

2) Rabenhorst, Kryptogamenflora, 4. Band, 2. Lief. 1885, pag. 124

3) a. a. 0. pag. 32 .

4) a. a. 0. pag. 112 , 
Die Neigung, nicht nur das Lumen der Chlorophyllzellen in die Mitte des Blattquerschnitts zu rücken, sondern auch die ganzen Zellen zu centriren, tritt uns in der Cymbifolium-Gruppe in auffallender Weise entgegen. Hier finden wir (abgesehen von Sph. Austini Sulliv.) an den Blättern derselben Pfląnze so beträchtliche Schwankungen in Betreff der Lagerungs- und Formverhältnisse der Chlorophyllzellen, dass von der rein centrischen Lage und Linsenform alle denkbaren Uebergänge zu der lateralen Lage und Dreieckform zu finden sind. Wenn wir bedenken, dass in der Jugend die Chlorophyllzellen, mögen sie später dem einen oder anderen Typus angehören, durchaus gleich gebildet sind, so ist es verständlich, dass Zwischenformen vorkommen können, es ist eben in der genannten Gruppe das Stellungsverhältniss noch nicht so fixirt wie bei Sph. rigidum und aculeatum, daher ist es unżulässig auf diese noch sehr wandelbaren Verhältnisse hin eine Artentrennung vorzunehmen wie es $\mathrm{Limpricht}$ gethan, der sein Sph. medium auf Stellung und Form der Chlorophyllzellen begründet.

Kehren wir zurück zur Betrachtung des Antheils der Chlorophyllzellen an der Aussteifung des Blattes.

Berücksichtigen wir den Umstand, dass die Stengelblätter von $s p h$. Girgensohnii m.; trotzdem dass die Hyalinzellen fast gänzlich resorbirt sind, eine beträchtliche Steifigkeit besitzen, welche letztere also nur von den Chlorophyllzellen herrührt, und dass bei anderen Arten die Stengelblätter mit faserlosen Hyalinzellen auch nicht der Steifigkeit entbehren, so dürfen wir annehmen, dass auch in den Astblättern (mit Ausnahme von Sph. rigidum, aculeatum und medium) es die Chlorophyllzellen sind, welche gleichsam das feste Gerüst darstellen, welches als Träger dem complicirt gebauten Wassersaugungs- und Speicherungs-System dient. Die einzelnen Elemente des letzteren, die Hyalinzellen, sind der ihnen zugetheilten Aufgabe entsprechend, äusserst rationell construirt und. derart mit den assimilatorischen Gerüstzellen verbunden, dass sie sich gegenseitig unterstützend, dem ganzen Blatt erhöhte Festigkeit verleihen. In der oberen Hälfte der abstehenden Blätter wird die in höherem Maasse erforderliche Festigkeit dadurch erzielt, dass die Berührungs- oder Verwachsungsflächen zwischen Chlorophyll- und Hyalinzellen viel grössere sind als in der unteren, bedeckten Blatthälfte, die weniger fest construirt zu. sein braucht (vergl. Fig. 10, 11,12 mit $13,14,15$ oder 17,18 mit 20 u. 21). Noch auffallender ist der Unterschied in der Grösse der Berührungsflächen von beiderlei Zellen, wenn wir die obere Hälfte der Blätter abstehender Aeste mit den Blättern der herabhängenden Aeste vergleichen, wie leicht ersichtlich aus dem bisher Mitgetheilten und den betreffenden Abbildungen.

Versuchen wir es jetzt noch

\section{die morohplogischen Verhältnisse des anatomischen Baus}

im Interesse der S y stematik zu verwerthen, sind doch vorliegende Untersuchungen zunächst nur im Interesse der Systematik unternommen worden und 
haben sich die mitgetheilten Entdeckungen und Reflexionen, gewissermaassen, beiläufig ergeben.

Wenn wir nochmals die dargelegten anatomischen Verhältnisse der :Sphagnumblätter überblicken, so tritt uns wie auch anderwärts im Pflanzenreich die Thatsache entgegen, dass je nach dem Bedürfniss, d. h. je nach den Anforderungen, welche an die physiologischen Leistungen eines Organs gestellt sind, der anatomische Bau abändert, dass aber bei allen Abänderungen doch etwas, wenigstens relativ, Stabiles übrig bleibt, das sich unserem Verständniss durchaus entzieht, weil es unabhängig von äusseren, oder überhaupt uns wahnehmbaren, Bedingungen ist. Es ist das Morphologische oder Morphotische, das Geheimniss der Form.

Es ist leicht einzusehen, dass es zweckmässig ist, wenn z. B. die Hyalinzellen in der oberèn Hälfte, zumal in der Spitze der Blätter abstehender Aeste anders gebaut sind als die in der unteren Blatthälfte oder wenn die der hängenden Aeste wieder anders construirt sind, aber wir sehen nicht ein, warum bei gewissen Formen die im Querschnitt dreieckigen Zellen sich von der Innenfläche des Blattes her, bei anderen von der Aussenfläche her zwischen die Hyalinzellen einkeilen, warum bei einigen die linsenförmigen Chlorophyllzellen genau in der Mitte; bei anderen etwas ausserhalb der Mitte zwischen den Hyalinzellen stehen, da doch in allen Fällen der gleiche Zweck erreicht wird. Wir müssen das als eine nicht weiter erklärbare Thatsache hinnehmen, denn wenn wir behaupten diese Eigenthümlichkeiten sind Folge der Vererbung, so haben wir damit im Grunde Nichts erklärt. Wir gewinnen aber für die Beurtheilung verwandtschaftlicher Beziehungen in diesen morphologischen Verhältnissen, wenn mit ihnen andere Eigenthümlichkeiten zusammenfallen und sich von Generation zu Generation im gleichen Zusammenhange vererben, ein sehr bedeutungsvolles Moment. Wir dürfen hiernach schliessen, dass alle Formen, welche in Bezug auf Form und Lagerung der Chlorophyllzellen und $\mathrm{g}$ l $\mathrm{e}$ i $\mathrm{chz}$ e it i g in gewissen anderen Eigẹnthümlichkeiten übereinstimmen, zu einem engeren Verwandschaftskreise géhören. Wollten wir nach der Gestalt and Lagerung der Chlorophyllzellen allein die gleichen Formen zusammenstellen, so würden wir Heterogenes vereinigen und Verwandtes trennen.

Wenn wir nun sämmtliche anatomisch-morphologischen Verhältnisse berücksichtigen, so kommen wir zu folgender Gruppirung, in der wir für die einzelnen Gruppen und Untergruppen (der Kürze und Uebersichtlichkeit wegen) das besonders characteristische Merkmal a 11 e in namhaft machen.

I. Stengel-Rindenzellen sehr gross, mit Fasern und Poren.

II. $\quad \because \quad$ relat. klein, ohne Fasern, mit oder ohne Poren.

Man könnte die erste Abtheilung als faserrindige (inophloea), die zweite als glattrindige (litophloea) bezeichnen. 
Die erste Abtheilung fällt mit der natürlichen Gruppe der c y m bi f o li a oder p a l u stri a zusammen.

In der zweiten Abtheilung können wir, unter Berücksichtigung der mittleren Blätter der abstehenden Aeste, nach der Gestalt und Lagerung der Chlorophyllzellen unterscheiden:

1) Chlorophyllzellen gleichschenklig oder gleichseitig dreieckig (triplagia). a. " $\quad$ an der Innenfläche des Blattes (endopleura).

2)

b. $\quad$,

a.

b.,
$" \quad$ "Aussenfläche $" \quad, \quad$ (exopleura).

linsen- oder tonnenförmig (diplagia).

von den hyalinen ganz umschlossen (perikleista).

nicht umschlossen (akleista).

In der Unterabtheilung 1, b ergeben sich wiederum zwei Untergruppen : $\alpha$ Chlorophyllzellen breit bis sehr breit, mit dreieckigem Lumen, ohne Papillen und $\beta$ Chlorophyllzellen schmal bis sehr schmal mit fast linsenförmigem und centrischem Lumen, mit Papillen.

Stellen wir nun die Gruppen und Untergruppen zusammen, sie mit den hergebrachten Namen bezeichnend, mit Aufführung der zu den betreffenden Gruppen g'ehörenden Arten, so ergiebt sich folgende Uebersicht.

\section{I nophloe a.}

1. Pa lustria (cymbifolia)

a. variabilia.

Sph. palustre $L$.

subspecies:

1. cymbifolium (Ehrh).

2. intermedium $\mathrm{m}$.

3. medium (Limpr).

4. papillosum (Ldbg).

b. pectinata.

Sph. imbricatum (Hornsch) m. (= Sph. Austini Sulliv).

II. Litophloea.

A. Triplagia.

a. Endopleura.

2. A cutifolia.

a. porosa.

Sph. fimbriatum Wils., Girgensohnii m., Russowii Warnst.

ß. tenella.

Sph. Warnstorfi m., tenellum Klingg., fuscum Klingg.

$\gamma$. deltoidea (oxyphylla).

Sph. quinquefarium (Braith) W., subnitens W. \& R., auctifolium Ehrh. ex parte. 
o. sulcata.

Sph. molle Sulliv. (= Sph. Mülleri Schimpr.)

b. Exopleura.

3. P a pill los a (squarrosa).

x. megalophylla.

Sph. squarrosum Pers., Sph. teres Ängstr.

ß. microphylla.

sph. Wulfianum Girg.

4. Cuspidata.

a. laciniata.

Sph. Lindbergii Schmpr.

$\beta$. erosa.

Sph. riparium Änstr.

$r$. triangularia.

Sph. cuspidatum Ehrh. ex. p.

subspecies ( $4-5$ ?)

o. tenerrima.

Sph. molluscum Bruch.

B. Diplagia.

a. Pericleista.

5. Rigida.

Sph. rigidum schmpr.

b. Acleista.

6. $\operatorname{Trun}$ c at a.

Sph. Angstroemii Hartm.

7. Cavifolia.

sph. cavifolium Warnst.

subspecies.

heterophylla:

1. subsecundum (N. a.. E.).

2. laricinum (Spruce).

hemiisophylla :

3. contortum (Schltz).

4. platyphyllum (Warnst.)

In einer kürzlich publicirten, kleinen Abhandlung ${ }^{1}$ ) habe ich sechs Gruppen unterschieden, aber zu einer dieser Gruppen, der der Truncata, bemerkt, dass die drei hier vereinigten Arten: Sph. Mülleri, rigidum und Angstroemii so verschieden seien, dass man in jeder den Typus einer besonderen Gruppe erblicken dürfe; demnach stellt sich ergentlich die Zahl dieser Gruppen in jener

1) Sitzungsber. der Dorpat Naturforschergesellschaft. October-Sitzung. 
Eintheilung auf a $\mathrm{cht}$ heraus. Sph. Mülleri Schmpr. habe ich hier zu den Acutifoliis gebracht, was bereits vor mir W a r n sto $\mathrm{rf}^{2}$ ) gethan, nicht nur wegen der Form und Lagerung der Chlorophyllzellen, sondern auch wegen Uebereinstimmung in der ganzen Configuration des Zellnetzes der Astblätter. Freilich weicht es von den übrigen Gruppengenossen in mancher Beziehung beträchtlich ab, so dass ihm eine Sonderstellung in der Acutifolium-Grunpe zukommt, doch schliesst es sich hier enger als irgend einer anderen Gruppe an. Mit Bezug auf die bekannte Randfurche der Astblätter nenne ich die Abtheilung Sulcata, ohne damit besonderes Gewicht auf diese Erscheinung legen zu wollen.

sph. rigidum steht in mehrfacher Beziehung ganz einzig unter den Gattungsgenossen da, woher die Stellung, welche es hier gefunden wohl gerechtfertigt erscheinen möchte. Auch Sph. Ångstroemii, das ich leider nie lebend an seinem Standort beobachtet, scheint mir einen besonderen Gruppen-Typus zu repräsentiren, der die nächsten Verwandten in der Gruppe Cavifolia mir zu finden scheint.

Die Abgrenzung der Arten innerhalb der unterschiedenen 7 Gruppen wollen wir hier nur soweit in's Auge fassen, als sie auf rein anatomischen Merkmalen beruht. Demnach haben wir es hier nur mit drei Gruppen zu thun, den Cuspidatis, Cavifoliis und Cymbifoliis, denn in der Acutifolium- und PapillosumGruppe sind Form und Grösse der Stengelblätter in erster Linie maasgebend, die Rigida und Truncata sind monotypisch und fallen daher aus dieser Betrachtung heraus.

Bei den Cuspidatis sind zwei Arten, Lindbergii und riparium durch ihre Stengelblätter dermaassen characterisirt, dass sie hier nicht in Betracht kommen, molluscum ist durch seine Chlorophyllzellen. (Fig. 29) und die Form der Astblätter leicht kenntlich. Bleibt also nur das alte cuspidatum Ehrh., das neuerdings mehrfach gespalten worden und zwar auf Grundlage des anatomischen Baus der Rinde, mit Berücksichtigung der Form und Grösse der Stengelblätter, der An- und Abwesenheit von Fasern in letzteren. Den Bau der Rinde wird man nach Untersuchung genügend zahlreichen Materials keineswegs als maassgebend anerkennen können, da die Abgrenzung oder Nichtabgrenzung der Rinde gegen den Holzkörper sogar bei einem und demselben Exemplar vorkommt, ja derselbe Stengel-Querschnitt auf der einen Seite deutliche Abgrenzung, auf der entgegengesetzten Seite durchaus keine Abgrenzung der Rinde erkennen lässt. Auch in Betreff der übrigen Merkmale sind so zahlreiche Uebergangsbildungen vorhanden, dass eine Unterscheidung von Sph. récurvum, cuspidatum, obtusum, fallax, laxifolium etc. als A rte $\mathrm{n}$ durchaus unzulässig ist, widrigenfalls uns der Begriff „A r t", - als eine Formengruppe, die aus unter einander nach allen Richtungen verbundenen Gliedern zusammengesetzt, sich gegen eine zweite

1) Hedwigia, I886, Heft VI. Zwei Artentypen der Sphagna aus der Acutifolium-Gruppe. 
ähnliche Formengruppe scharf abgrenzt, - verloren gehen und die Systematik daher in einen chaotischen Zustand gerathen muss.

In der Subsecundum - Gruppe hat man sogar nach der Zahl der Rindenschichten, ob ein oder zwei bis mehrschichtig, zwei Artengruppen unterschieden, in jeder, je nach der Häufigkeit der Fasern in den Stengelblättern, je 2 Arten aufgestellt. Meine Untersuchungen haben mir gezeigt, dass die Zahl der Rindenschichten hier durchaus schwankend ist, dass es Formen giebt, die anderthalb Rindenschichten besitzen, insofern am halben Stengelumfang die Rinde aus zwei Zelllagen besteht. Es passt natürlich solch' eine Form weder in die eine noch andere Art, oder sie kann mit demselben Recht der einen wie der anderen zugezählt werden, zumal in Bezug auf die Stengelblätter auch nach allen Beziehungen Zwischenformen vorkommen. Ich kann daher in Sph. subsecundum, laricinum, contortum und platyphyllum nur Formen einer Art sehen und dieselben höchstens als „subspecies“ gelten lassen.

Was nun schliesslich die Cymbifolia betrifft, so hat man hier je nach der Lagerung und Form der Chlorophyllzellen und dem Vorhandensein von Papillen oder Kammfasern die alte Art in, vier neue gespalten: cymbifolium, medium, papillosum und Austini. Von Sph. glaucum Klingg. und subbicolor Hmpe. wollen wir hier ganz absehen, da ersteres eine durch Färbung und squarrose Blattbildung ausgezeichnete Form von Cymbifolium ist, während letzteres nur eine hemiisophylle Form darstellt.

Abgesehen von den Kammfasern, (die mitunter nur sehr spärlich ausgebildet sind) ist Sph. Austini durch die constante Form und Lagerung der Chlorophyllzellen so ausgezeichnet, dass wir keinen Augenblick über dessen Artenwerth im Zweifel bleiben können. Dagegen finden wir bei den drei anderen Arten in Bezug auf Form und Lagerung der Chlorophyllzellen wie Ausbildung von Papillen solch' eine Variabilität, dass es schlechterdings 'unmöglich ist auf Grundlage genannter Merkmale Arten zu begründen. Dazu kommt, dass in Bezug auf Rindenbildung (d. h. Häufigkeit der Faserung und Perforation der Rindenzellen), Grösse, Form und Faserung der Stengelblätter keine festen Anhaltspunkte zu gewinnen sind, weil auch hier nach allen Richtungen hin Schwankungen vorkommen.

In Bezug auf Form und Stellung der Chlorophyllzellen sind die Blätter in der Cymbifolium-Gruppe in Umformung begriffen, es hat sich in dieser Beziehung noch kein stabiler Zustand herausgebildet. Man vergleiche unter einander die Figg. 55, 56 und 57; sie sind nach Querschnitten gezeichnet, die von Blättern derselben Pflanze stammen und etwa in derselben Region die Blätter getroffen. Ebenso Fig. 52 und 53, denen ich noch eine dritte hätte hinzufügen kőnnen mit durchaus centrischer Lage und linsenförmiger Gestalt der Chlorophyllzéllen, von Blättern derselben Pflanze einer papillösen Form. Zeichnungen ron Blattquerschnitten des Sph. medium Limpr. konnte ich nicht mehr auf 
den Tafeln unterbringen, ich liess sie fort im Interesse anderer, da die typische Lagerung der Form der Chlorophyllzellen hier.gleichkommt der in Fig. 63 dargestellten, und die Uebergangsformen den in Fig. 56 und 57 dargestellten Lagerungs- und Formverhältnissen entsprechen. Im Ganzen selten habe ich an Limprichts medium, bei centrischer Lage der Chlorophyllzellen, die Convexität der Hyalinzellen an beiden Blattflächen gleich gefunden; meist ist die Wölbung an der Blatt-Aussenfläche stärker, bis sehr viel stärker als an der Innenfläche und damit hängt auch die Verdickung der Membranstücke zwischen dem Lumen der Chlorophyllzellen und der Blattoberfläche zusammen. Gar oft habe ich mir beim Durchmustern der Blatt-Querschnitte einer Cymbifolium-Form am Ende die Frage stellen müssen: wohin ist wohl dieses Sphagnum zu bringen, zu cymbifolium oder medium? Der Mehrheit der Querschnittsansichten nach entscheide ich mich für medium. Nach einiger Zeit habe ich dies elbe Pflanze nochmals untersucht und habe mich dann veranlasst gesehen mich für cymbifolium zu entscheiden! Ich denke, da hört jede Artunterscheidung auf.

Dasselbe gilt von den Papillen. Hat man Blätter mit stark ausgeprägten Papillen vor Augen, so möchte man nicht einen Augenblick anstehen, hierin ein treffliches Art-Unterscheidungsmerkmal zu erblicken. Wenn man aber auf Formen stösst, die nur hie und da in der unteren Blatthälfte spärlich gesäete Papillen aufweisen oder die Papillen so klein werden, dass man zweifelhaft bleibt ob man es noch überhaupt mit Papillen zu thun hat, so steht es, wird wohl jeder Unbefangene gestehen, gar misslich mit der Artunterscheidung. Wollte man ferner der Lagerung und Form der Chlorophyllzellen specifischen Werth zuerkennen, so müsste man das Sph. papillosum Lindbg. wiederum in zwei Arten spalten, denn es kommen hier rein centrische, linsenförmige und gestrecktdreieckige, an der Blattinnenfläche befindliche Chlorophyllzellen vor; daher erklären sich anch die bei versichiedenen Autoren sich widersprechenden Angaben in Bezug auf die Lagerungs- und Formverhältnisse der Chtorophyllzellen bei Sph. papillosum. Man müsste nach demselben Princip auch innerhalb der Squarrosa die nicht papillösen Formen (solche kommen vor) von den papillösen trenuen.

Alles in Allem kommen wir, wenn es sich um die Beurtheilung verwandtschaftlicher Verhältnisse handelt, in Bezug auf den anatomischen Bau, ebenso wie in Bezug auf äussere Gliederung, ' zu dem Schluss, dass wir hier wie dort morphologische und physiologische Dignität wohl zu unterscheiden haben, wollen wir uns nicht grober Nissgriffe schuldig machen. 


\section{Erklärung der Abbildungen.}

Sämmtliche Figuren sind mit Hülfe der Camera lucida mach Nachet gezeichnet worden. Fig. 2 und 9 sind mit Objectiv $V$ und Ocular 0 von Seibert, alle übrigen Figuren mit Objectiv VII (Immersion) und Ocular 0 von Seibert aufgenommen worden.

\section{Taf. I.}

Fig. 1. Einige Hyalin- und Chlorophyllzellen aus dem oberen Drittel eines Blattes abstehender Aeste von Sph. fuscum Klingg . bei mittlerer Einstellung auf die Hyalinzellen. Cl. Chlorophyllkőrner; a gefurchteter Innenrand der Ringplatte; b Furche in der Aufsicht; $p$ ein umwalltes Loch.

Fig. 2. Stück des Zellnetzes aus der oberen Blatthälfte (abst. Aeste) von Sph. Warnstorfi $m$. von der Aussenfläche gesehen.

Fig. 3. Stuck einer Hyalinzelle von Sph. rigidum Schmpr. in der Aufsicht; die punktirten Linien deuten die Ausdehnung und den Verlauf der, ausserhalb des Focus liegenden, Chlorophyllzellen an. a Wulst des Meniscusrandes im optischen Querschnitt; p Porus; q Queranastomosen der Fasern.

Fig. 4. Stücke dreier Hyalinzellen von Sph. aculeatum Warnst. Bedeutung der Buchstaben wie in voriger Figur.

Fig. 5. Parallel zur Oberfläche (längs-)durchschnittene Hyalin- und Chlorophyllzellen von sph. Warnstorfii m.

Fig. 6. Stück einer Hyalinzelle von Sph. imbricatum (Hornsch.) m. die „Kammfasern" KF. zeigend in der Ausdehnung der Chlorophyllzellen, deren nach oben gerichtete Kante dem Beschauer zugekehrt ist.

Fig. 7. Stück eines Querschnitts durch das Blatt von Sph. Russowii W., in der oberen Hälfte des Blattes. Bei a abgeschnittener Rand der Ringplatte.

Fig. 8. Stück einer Hyalinzelle mit Chlorophyllzellen aus dem Blatte von 'Sph. cyclophyllum F. L. Bedeutung der Buchstaben wie in Fig. 3 u. 4.

Fig. 9. Stück des Zellnetzes aus dem unteren Theil eines Stengelblattes von Sph. teres Angstr. die Querfältchen, F, zeigend.

\section{Taf. II.}

Der Kürze halber bediene ich mich im Folgenden einiger Abkürzungen. A Blatt bedeutet Blatt eines abstehenden Astes. H Blatt das eines herabhängenden Astes. 0 1/3 oberes. Drittel; 0 1/2 obere Hälfte; U 1/2 untere Hälfte des betreffenden Blattes. Bis auf Fig. 16 und 35 stellen alle übrigen Stücke von Querschnitten durch Blätter dar.

Fig. 10. Sph. Warnstorfii m. A Blatt, 0 1\%3. a gespreizter Wulst der Ringplatte Rp, b mittlerer ringförmiger Vorsprung der Lochwandung. 
Fig. 11. Sph. Russowii W. A Blatt, 0 1/3.

Fig. 12. Sph Girgensohnii m. A Blatt $0 \frac{1 / 3}{3}$.

Fig. 13. Sph. fimbriatum Wils. A Blatt, U 1/2, bei a abgeschnittene Ränder der Ringplatte.

Fig. 14. Sph. Girgensohnii m. A Blatt, U 1/2.

Fig. 15. Sph.Warnstorfi m. A Blatt, U 1/2. Hyalinzellen von der Mitte zum Rande beträchtlich an Grösse zunehmend.

Fig. 16. Sph. Girgensohnii m. Stück des Zellgewebes aus der oberen Hälfte eines Stengelblattes. Pf. Pseudofasern; bei F eine wirkliche Faser auf der Pseudofaser.

Fig. 17. Sph. subnitens $W . \& R$. A Blatt nicht weit unterhalb der Spitze getroffen.

Fig. 18. Sph. quinquefarium (Braith) W. A Blatt, 0 1/3.

Fig. 19. Sph. acutifolium Ehrh. A Blatt, 0 1/2.

Fig. 20. Sph. subnitens, A Blatt, U 1/2.

Fig. 21. Sph. Mülleri Schmpr. A Blatt: 0 1/2.

Taf. III.

Fig. 22. Sph. riparium Angstr. A Blatt, 0 1/3.

Fig. 23. $" \quad$ A Blatt, $0 \frac{1}{2}$.

Fig. 24. $"$ H Blatt, U $1 / 2$.

Fig. 25. Sph. cuspidatum Ehrh. v. mollissimum m. A Blatt, 0 1/2.

Fig. 26. Sph. cuspidatum Ehrh., grosse Wasserform. A Blatt 0 1/2.

Fig. 27. Sph. riparium, A Blatt, $0 \mathrm{1} / 2$.

Fig. 28. Sph. recurvum P. d. B. A Blatt, Mitte.

Fig. 29. Sph. molluscum Bruch. A Blatt, Mitte.

Fig. 30. Sph. Lindbërgii Schmpr. A Blatt, 0 1/2.

Fig. 31. Sph. subsecundum v. contortum, A Blatt, 0 1/2.

Fig. 32. Sph. cribrosum Lindbg. A Blatt, U 1/2.

Fig. 33. Sph. Pylaiei Brid. A Blatt, U 1/2.

Fig. 34.

Fig. 35 .

Clz. Chlorophyllzelle.
A Blatt, $01 / 3$.

Hyalinzelle in der Aufsicht. F. Falten in der Membran.

\section{Taf. IV.}

Fig. 36. Sph. squarrosum Pers. A Blatt, 0 1/3.

Fig. 37. $", \quad " \quad .0 \frac{1 / 2}{2}$.

Fig. 38. " $", \quad$ U $1 / 2$.

Fig. 39. Sph. teres" Angstr. $\quad " \quad$ U $1 \frac{1}{2}$.

Fig. $40 . \quad " \quad " \quad 0 \quad 1 \frac{1}{2}$.

Fig. 41. " $\quad " \quad 0 \quad 1 \frac{1}{3}$.

Fig. 42. Sph. Wulfianum Girg. A Blatt, 0 1/3.

Fig. $43 . \quad " \quad, \quad 0 \quad \frac{1}{3}$.

Fig. $44 . \quad " \quad \therefore \quad, \quad 0 \quad 1 / 2$.

Fig. $45 . \quad " \quad \quad " \quad$ U $1 / 2$.

Fig. 46. $" \quad H$ Blatt U $1 / 2$.

Fig. 47. $\quad " \quad$ A Blatt, U $1 / 2$ 
Fig. 48. Sph. Ångstroemii Hartm. A. Blatt, 0 1/3. Eig. 49.

Fig. 50.

Fig. $5 \mathrm{l}$.

$\begin{array}{lll}" & \text { U } 1 / 2 \\ " & U & 1 / 2 \\ " & 0 & 1 / 2\end{array}$

\section{Taf. V.}

Fig. 52 u. 53. Sph. papillosum Lindbg. A Blatt, U 1/2.

Fig. 54. Simbricatum (Hornsch) m. A Blatt, U. 1/2.

Fig. 5̄.̦-57. Sph. cymbifolium Ehrh. A Blatt, Mitte.

Fig. 58. Sph. rigidum Schmpr. A Blatt, Mitte.

Fig. 59.

$\mathrm{H}$ Blatt, U $1 / 2$.

Fig. 60 u. 61.

A Blatt, Mitte.

Fig. 62. Sph. cyclophyllum S. L. A Blatt, U ${ }^{1}{ }_{2}$.

Fig. 63. Sph. aculeatum Wernst. A Blatt, Mitte. 


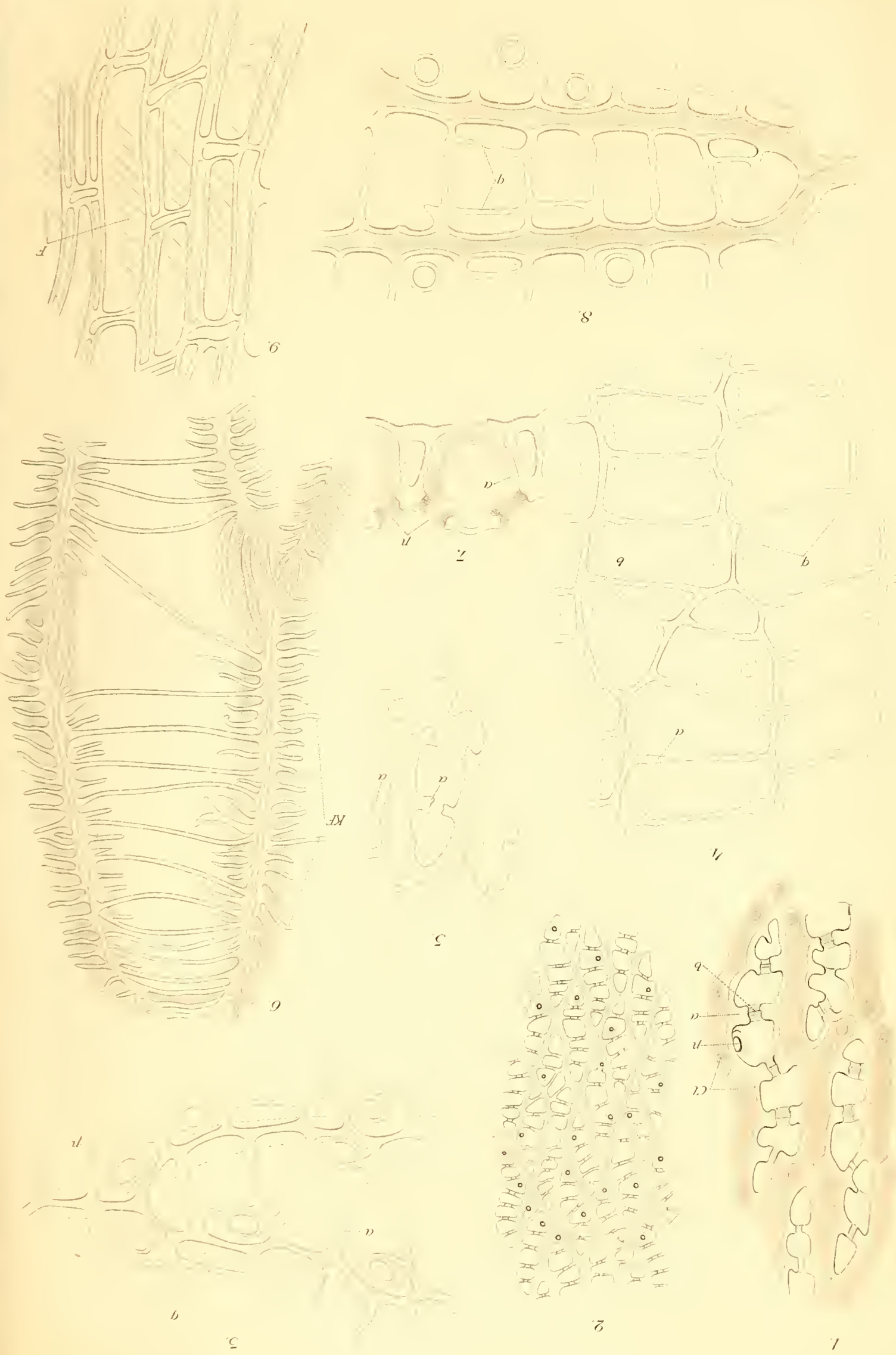



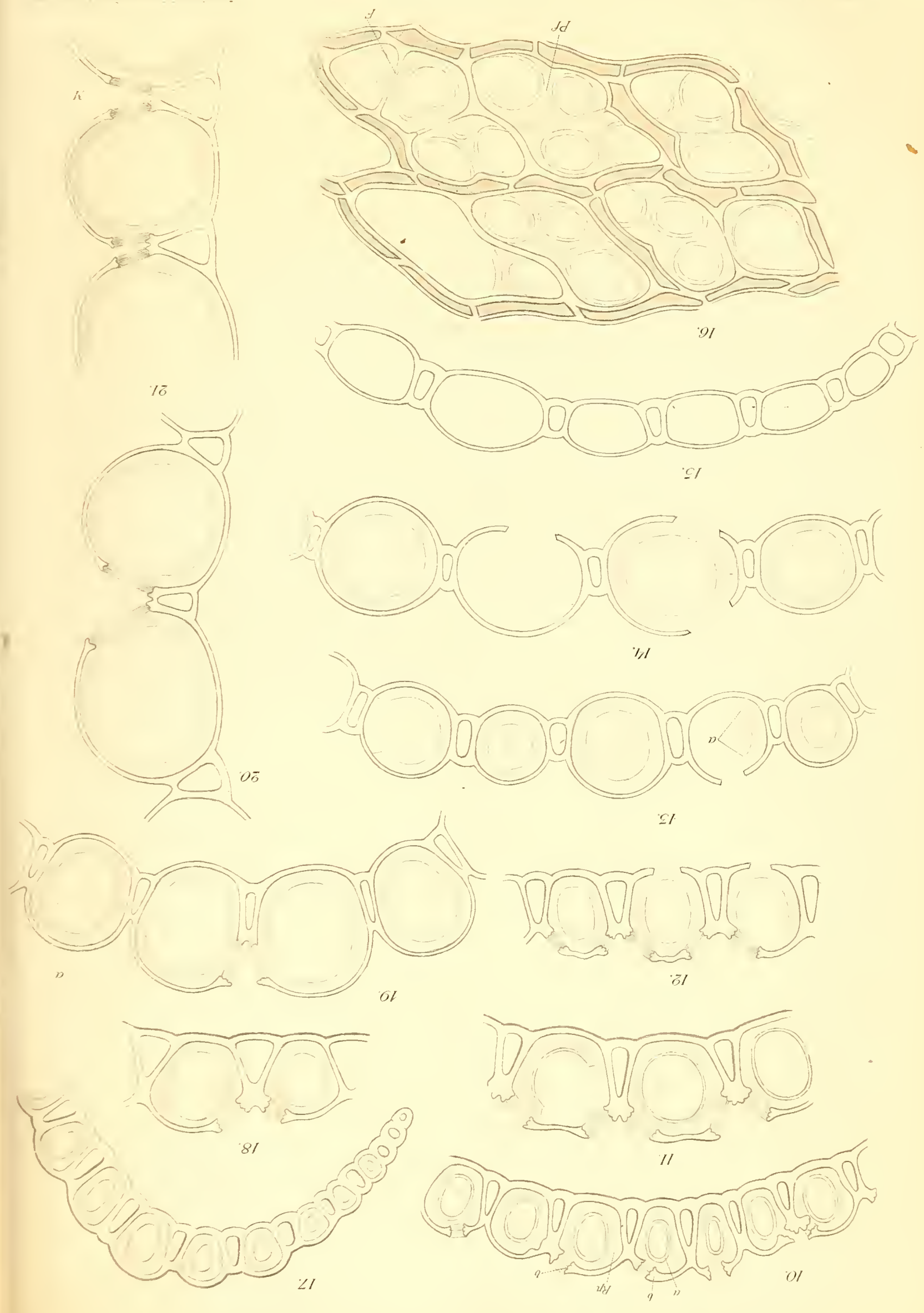





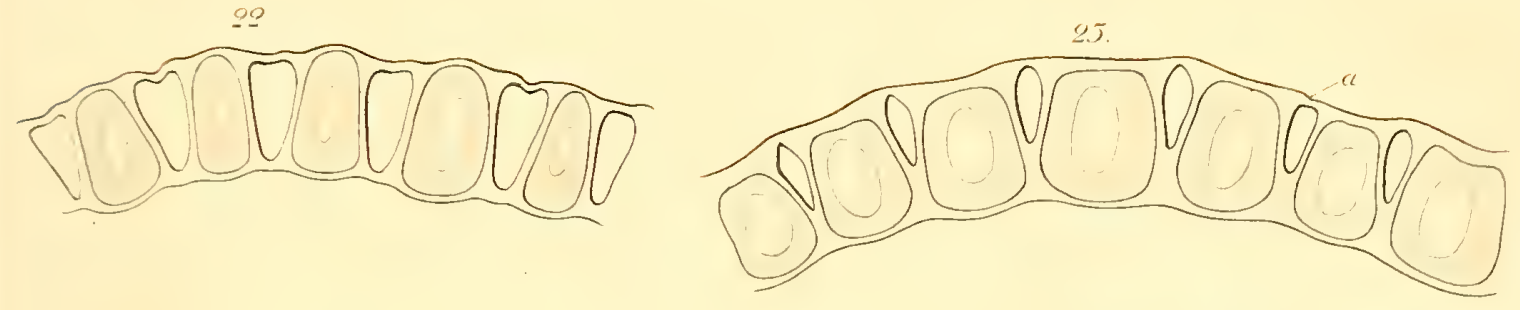

24.

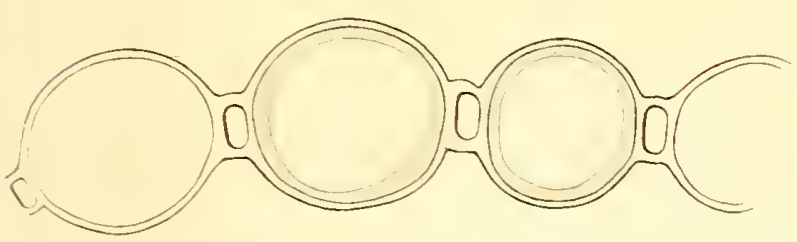

\section{0}

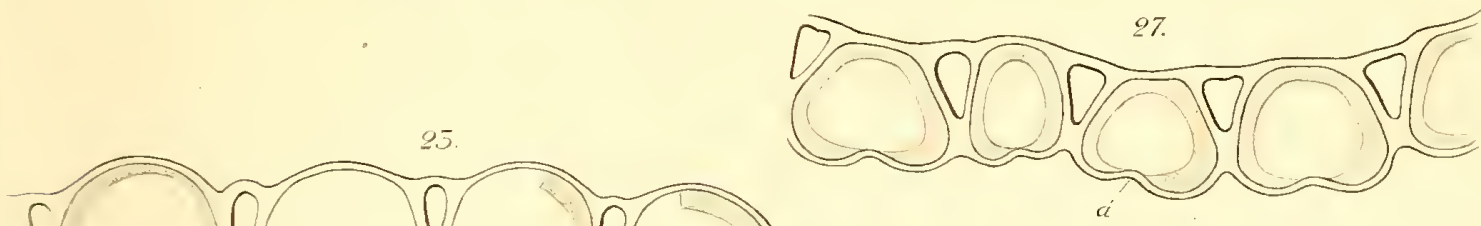

(1)
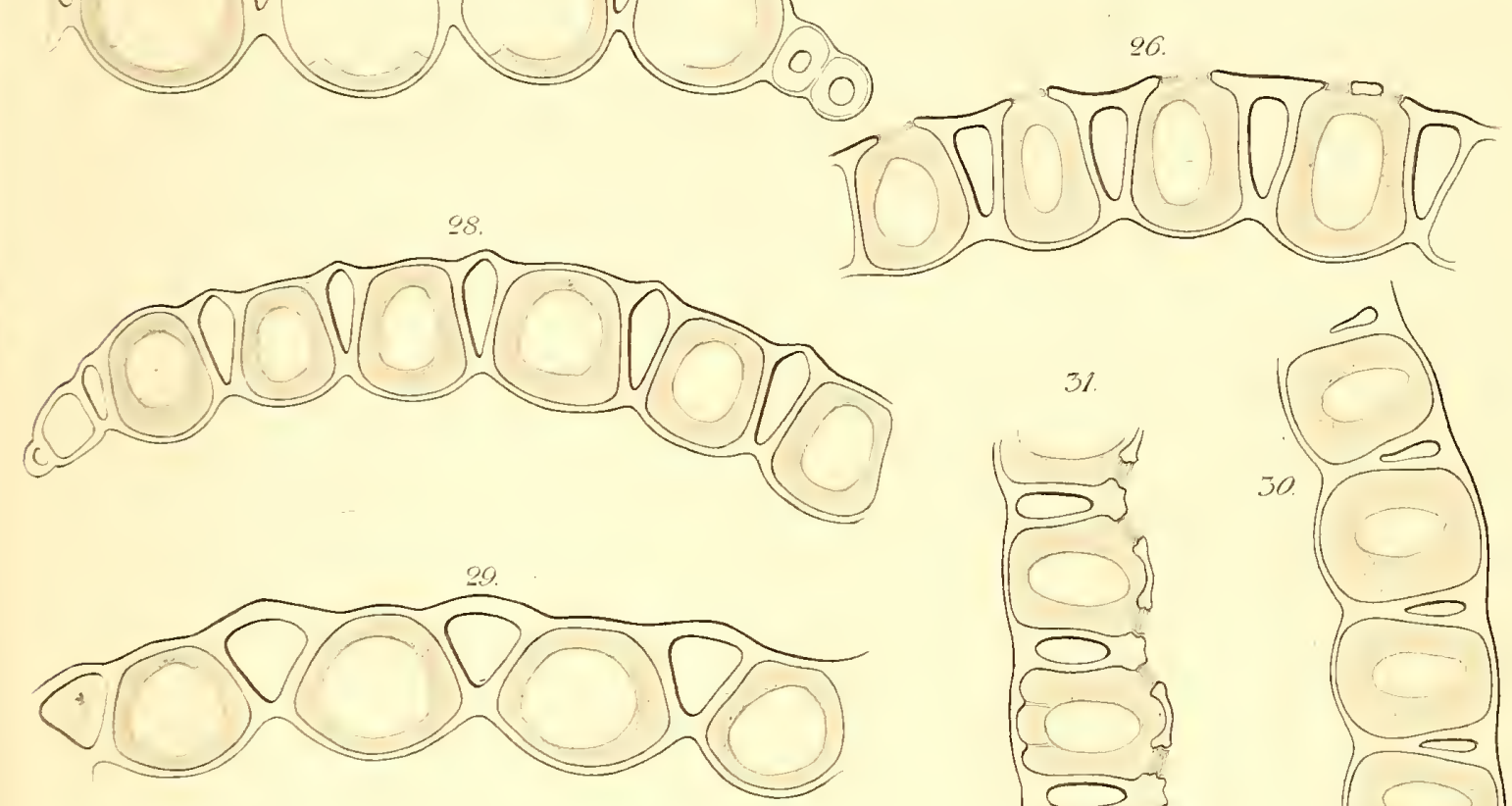

32.

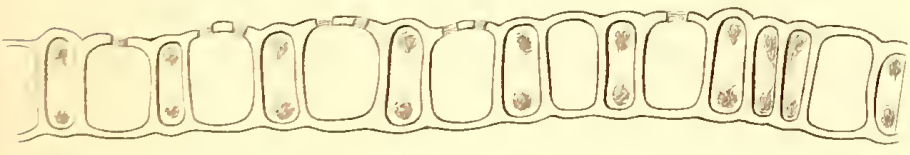
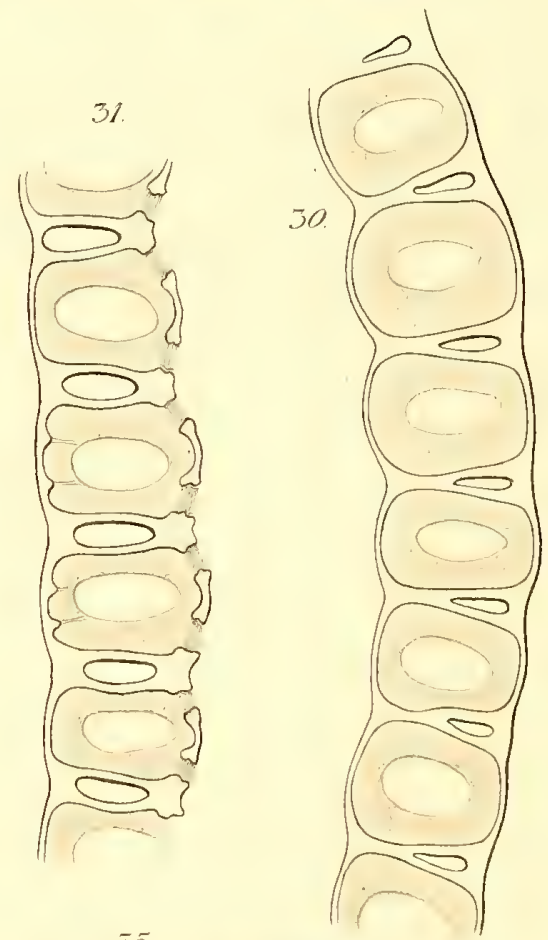

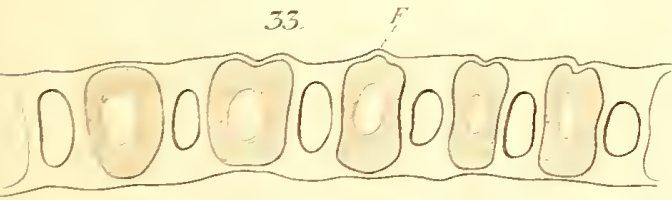



U.

$5 \pi$
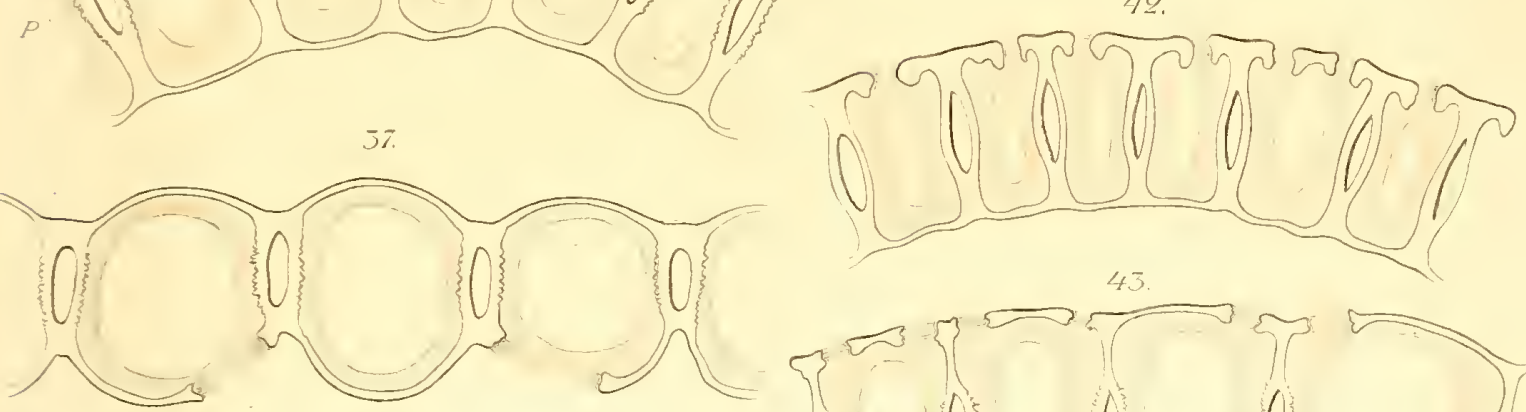

$\left\{\begin{array}{l}0 \\ 0\end{array}\right.$
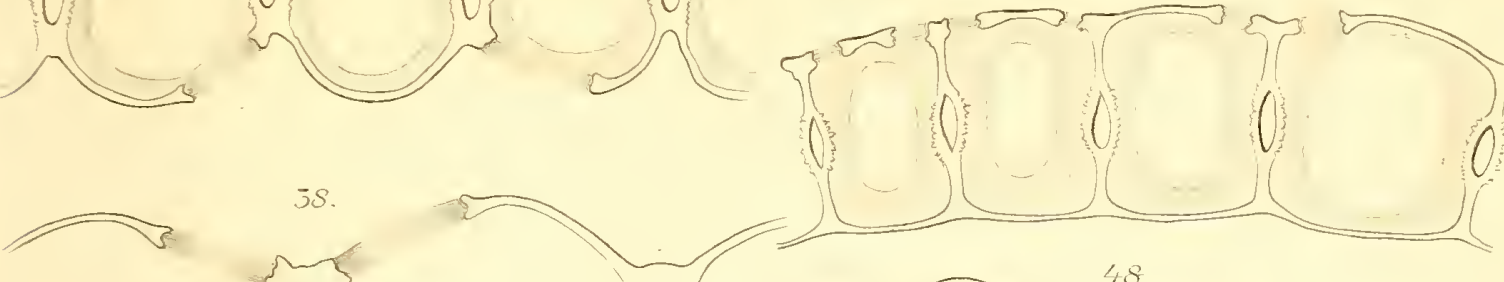

48.
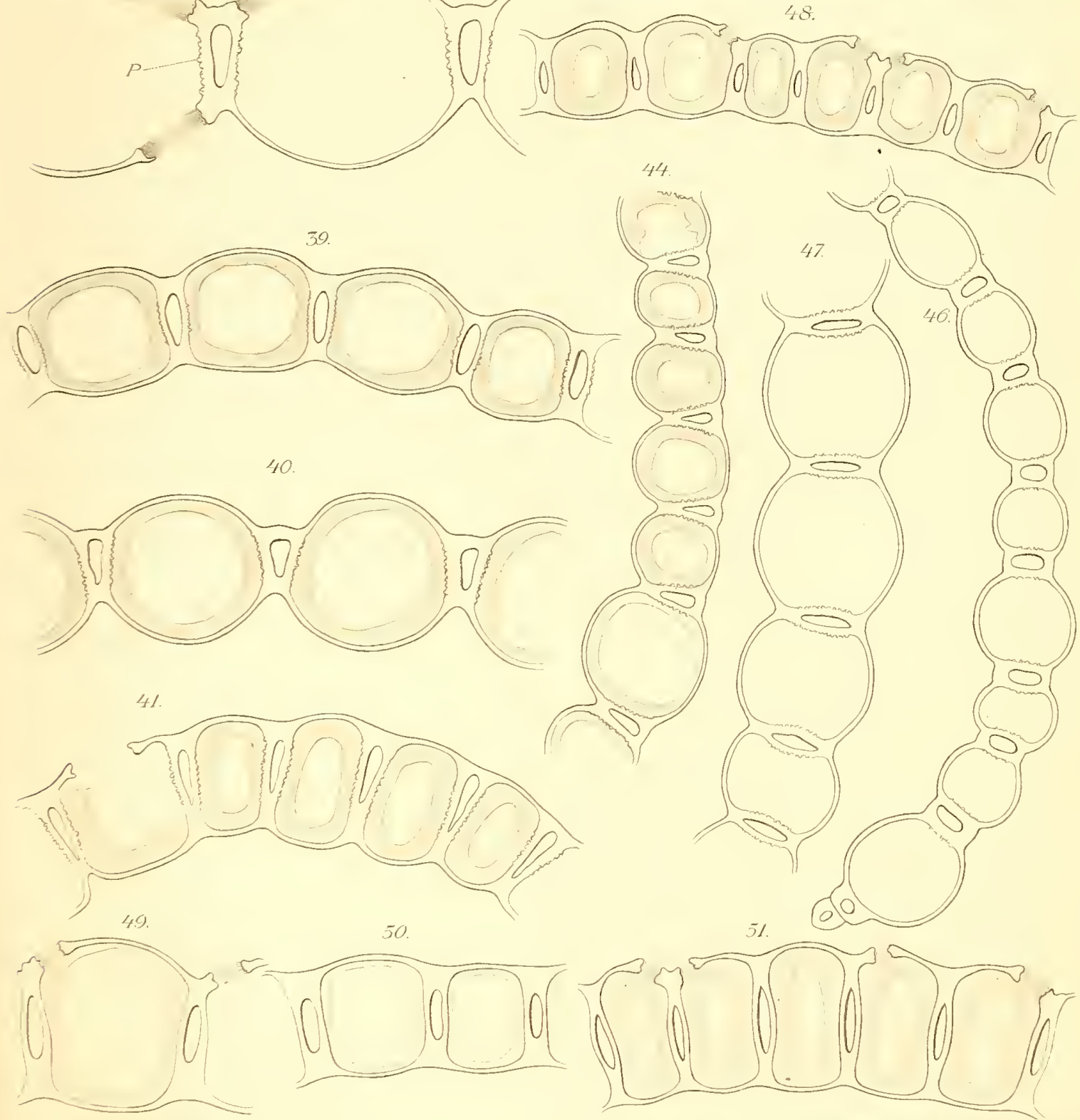


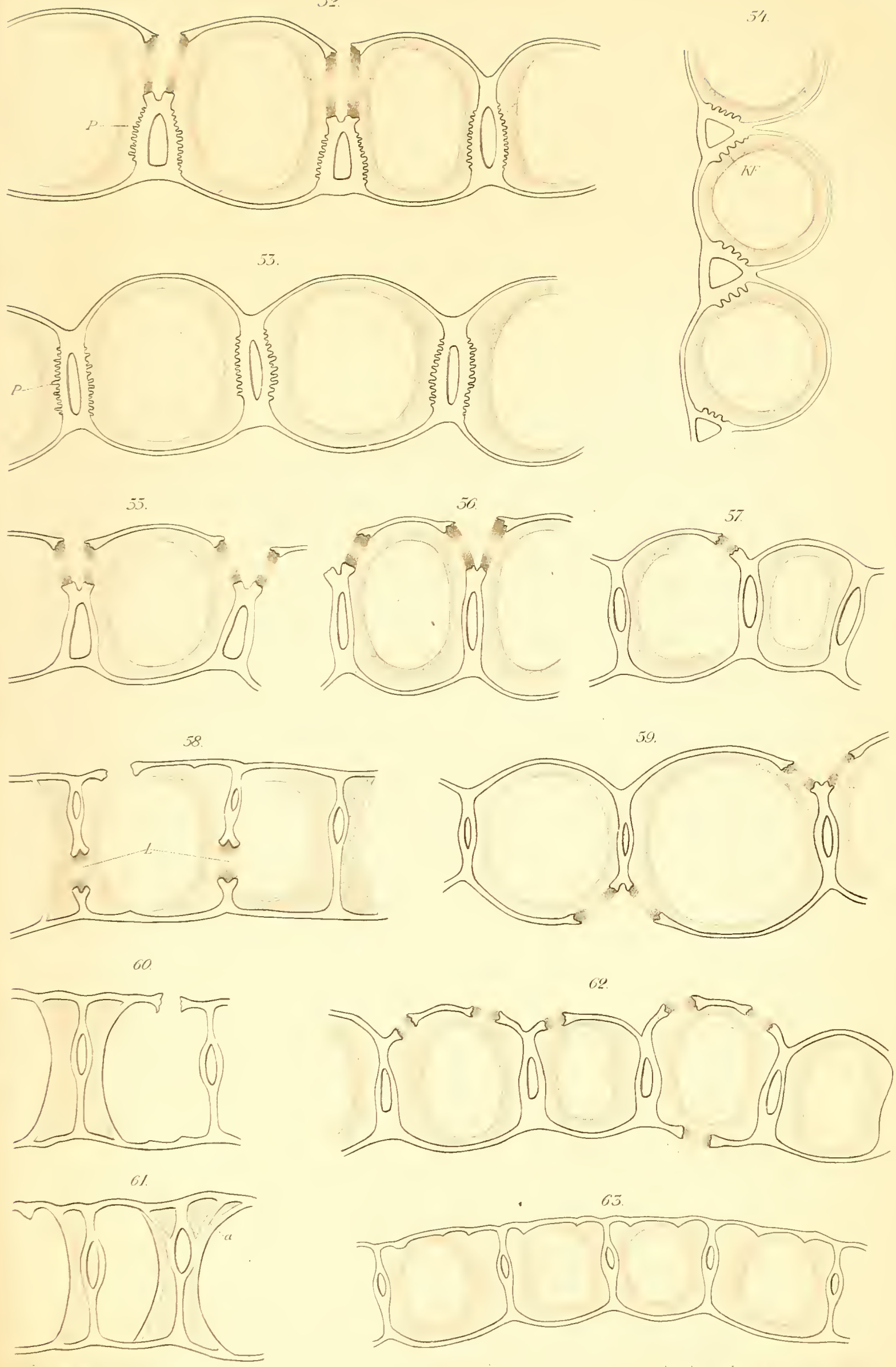




SMITHSONIAN INSTITUTION LIBAARIES

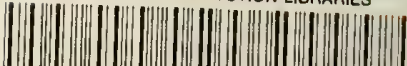
$\begin{array}{llll}3 & 9088 & 00596 & 3954\end{array}$ 